\title{
A novel Perceptual Two Layer Image Fusion using Deep Learning for Imbalanced COVID-19 Dataset
}

\author{
Omar M Elzeki ${ }^{\text {Corresp., Equal first author, } 1 \text {, Mohamed Abd Elfattah }}{ }^{\text {Equal first author, } 2 \text {, Hanaa Salem }}{ }^{\text {Corresp., } 3}$, Aboul Ella Hassanien ${ }^{4,5}$, \\ Mahmoud Shams ${ }^{6}$ \\ 1 Faculty of Computers and Information Sciences, Mansoura University, Mansoura, Egypt \\ 2 Misr Higher Institute for Commerce and Computers, Mansoura, Egypt \\ 3 Communications \& Computer Engineering Department, Faculty of Engineering, Delta University for Science \&Technology, Gamasa, Egypt \\ 4 Faculty of Computers and Artificial Intelligence, Cairo University, Cairo, Egypt \\ ${ }^{5}$ Scientific Research Group in Egypt(SRGE), Cairo, Egypt \\ ${ }^{6}$ Faculty of Artificial Intelligence, Kafrelsheikh University, Kafrelsheikh, Egypy \\ Corresponding Authors: Omar M Elzeki, Hanaa Salem \\ Email address: omar_m_elzeki@mans.edu.eg, hana.salem@deltauniv.edu.eg
}

Background and Purpose: COVID-19: is a new strain of viruses that causes life stoppage worldwide. Up till now, the new coronavirus COVID-19 is spreading rapidly across the world and poses a threat to people's health. Experimental medical tests and analysis have shown that the infection of lung occurs in almost all COVID-19 patients. Although Computed Tomography (CT) of the chest is a useful imaging method for diagnosing diseases related to the lung, chest X-ray (CXR) is more widely available, mainly due to its lower price and results. Deep learning (DL), one of the significant popular artificial intelligence techniques, is an effective way to help doctors analyze how a large number of CXR images is crucial to performance.

Materials and Methods: In this paper, we propose a novel perceptual two-layer image fusion using DL to obtain more informative CXR images for COVID-19 Dataset. To assess the proposed algorithm performance, the dataset used for this work includes 87 CXR images acquired from 25 cases, all of which were confirmed with COVID-19. The dataset preprocessing is needed to facilitate the role of (CNN). Thus, hybrid decomposition and fusion of Nonsubsampled Contourlet Transform (NSCT) and CNN_VGG19 as feature extractor was used.

Results: Our experimental results show that imbalanced COVID-19 datasets can be reliably generated by the algorithm established here. Compared to the COVID-19 Dataset used, the fused images have more features and characteristics. In evaluation performance measures, six metrics are applied, such as $\mathrm{Q}^{\mathrm{AB} / \mathrm{F}}$, $Q^{M I}$, PSNR, SSIM, SF, and STD, to determine the evaluation of various medical image fusion (MIF). In the $Q^{M I}$, PSNR, SSIM, the proposed algorithm NSCT+CNN_VGG19 achieves the greatest and the features characteristics found in the fused image is the largest. We can deduce that the proposed fusion algorithm is efficient enough to generate CXR COVID-19 images that are more useful for the examiner to explore patient status.

Conclusions: A novel image fusion algorithm using DL for imbalanced COVID-19 Dataset is the crucial contribution of this work. Extensive results of the experiment display that the proposed algorithm NSCT+CNN_VGG19 outperforms competitive image fusion algorithms. 


\title{
1 A novel Perceptual Two Layer Image Fusion using 2 Deep Learning for Imbalanced COVID-19 Dataset
}

3

4 5

6

\author{
O. M. Elzeki ${ }^{1}$, Mohamed Abd Elfattah ${ }^{2}$, Hanaa Salem³ ${ }^{3}$ Aboul Ella Hassanien ${ }^{4 *}$, Mahmoud. Y. \\ Shams ${ }^{5}$ \\ ${ }^{1}$ Faculty of Computers and Information, Mansoura University, 35516, Mansoura, Egypt \\ ${ }^{2}$ Misr Higher Institute for Commerce and Computers, Mansoura, Egypt \\ ${ }^{3}$ Communications \& Computer Engineering Department, Faculty of Engineering, Delta \\ University for Science \&Technology, Gamasa, Egypt. \\ ${ }^{4}$ Faculty of Computers and Artificial Intelligence, Cairo University, Egypt \\ ${ }^{5}$ Faculty of Artificial Intelligence, Kafrelsheikh University, Kafrelshiekh 33511, Egypt \\ *Scientific Research Group in Egypt (SRGE). Cairo, Egypt.
}

Corresponding Author:

Omar Mohamed Elzeki, Hanaa Salem Marie

Mansoura, Dakhlia, Egypt

Email address: omar_m_elzeki@mans.edu.eg_hana.salem@deltauniv.edu.eg

\section{Abstract}

Background and Purpose: COVID-19: is a new strain of viruses that causes life stoppage worldwide. Up till now, the new coronavirus COVID-19 is spreading rapidly across the world and poses a threat to people's health. Experimental medical tests and analysis have shown that the infection of lung occurs in almost all COVID-19 patients. Although Computed Tomography (CT) of the chest is a useful imaging method for diagnosing diseases related to the lung, chest X-ray (CXR) is more widely available, mainly due to its lower price and results. Deep learning (DL), one of the significant popular artificial intelligence techniques, is an effective way to help doctors analyze how a large number of CXR images is crucial to performance.

Materials and Methods: In this paper, we propose a novel perceptual two-layer image fusion using DL to obtain more informative CXR images for COVID-19 Dataset. To assess the proposed algorithm performance, the dataset used for this work includes 87 CXR images acquired from 25 cases, all of which were confirmed with COVID-19. The dataset preprocessing is needed to facilitate the role of $(\mathrm{CNN})$. Thus, hybrid decomposition and fusion of Nonsubsampled Contourlet Transform (NSCT) and CNN_VGG19 as feature extractor was used.

Results: Our experimental results show that imbalanced COVID-19 datasets can be reliably generated by the algorithm established here. Compared to the COVID-19 Dataset used, the fused images have more features and characteristics. In evaluation performance measures, six metrics are applied, such as $\mathrm{Q}^{\mathrm{AB} / \mathrm{F}}, \mathrm{Q}^{\mathrm{MI}}$, PSNR, SSIM, SF, and STD, to determine the evaluation of various medical image fusion (MIF). In the $\mathrm{Q}^{\mathrm{MI}}$, PSNR, SSIM, the proposed algorithm NSCT + CNN_VGG19 achieves the greatest and the features characteristics found in 
43 the fused image is the largest. We can deduce that the proposed fusion algorithm is efficient 44 enough to generate CXR COVID-19 images that are more useful for the examiner to explore 45 patient status.

46 Conclusions: A novel image fusion algorithm using DL for imbalanced COVID-19 Dataset is 47 proposed algorithm NSCT + CNN_VGG19 outperforms competitive image fusion algorithms.

Keywords: Coronavirus, COVID-19, NSCT, CNN, Image Fusion, Deep Learning, and VGG19.

\section{Introduction}

MIF provides crucial information representing the source images helpful in diagnosis, prognosis, treatment, and classification (Ganasala et al., 2016). For a quick and accurate diagnosis, supplementary information must be extracted from the various source images in one image. It is well known that medical images have different and variable modalities that carry information with complementary properties (Srivastava et al., 2016). A selection of image pixels or patches is performed to construct a fused image in the spatial domain to preserve each source image's information. The major limitation of spatial information fusion is the non-integrality of the fused information producing the contrast and sharpness, which in turn leads to the decrease of detailed information in the fused image (Liu et al., 2017; Zhu et al., 2018; and Zhiqin et al., 2019).

Chest X-Ray image, also called CXR is generally a common method and non-invasive radiology examination. In the recent pandemic of COVID-19, the use of CXR is desired because radiologists easily interpret it, and the time-consumption is decreased with minimum assessment errors (Liu et al., 2019). The fusion of CXR images can be performed in feature extraction and classification stages, as demonstrated by (Liu et al., 2019) and (Huang et al., 2020). Furthermore, CXR can be utilized as large-scale input images that can be combined with deep convolution neural networks $(\mathrm{DCNN})$ to boost the performance of the variable sizes of thoracic diseases $(\mathrm{Hu}$ et al., 2020)

One of the recent fusion techniques is fusion based on NSCT. The fusion technique can avoid spectral aliasing and provide more characteristics of the invariance translation (Bing et al., 2020). Moreover, NSCT is proposed in the input image decomposition level to transform the source image to both low and high-pass subbands, providing more details and reservation of input images (Liu et al., 2019). The limitation of using NSCT is the resulting fusion performance in humanoid visualization based objective metrics that need more enhancement like adding an optimizer or classifier (Bhatnagar et al.,2013; Tian et al., 2016; and Gomathi et al., 2016). Another fusion technique is the utilization of deep learning in fusion strategies. Liu et al. (Liu et al., 2018) present $\mathrm{CNN}$ for image fusion by which a weighted map of the source images are generated with promising results.

The correlation between NSCT and deep learning is demonstrated in (Liu et al., 2017) by which a fusion based on CNN and NSCT of multi-focus images is performed. Moreover, two-scale decomposition transforms are presented in (Lahoud \& Süsstrunk, 2019) such that the image layers are fused based on CNN intermediate feature maps. They used a guided filter to smooth the weight maps and enforce consistency with the source images. Therefore, the merge between deep learning and NSCT is very attractive and helpful in discovering more details and consuming a minimum of less time (Hermessi et al., 2018; Tang et al., 2018; Amin et al., 2019).

The development and design of an effective MIF algorithm based on DL is still an open area. The key contributions of this research may be summarized as the following: 
- Initially, the proposed approach decomposes the image into subbands using NSCT.

- For feature extraction of the output CXR COVID-19 images from NSCT, CNN-VGG19 is then utilized.

- Euclidean distance and weights subband calculations are applied to obtain the fused rules, a temporal consistency of extracted features, Euclidean distance, and weights subband calculations.

- A fused image is computed using the inverse NSCT.

- Finally, the comparative evaluation was performed using two methods; the first method is to determine the pre-trained framework efficiency using evaluation metrics. While the second method is based on classifying the fused CXR COVID-19 images using the deep learning approach CNN-VGG19 compared with the state-of-the-art.

The paper is organized as follows: in section 2, the authors review the research field's literature. Section 3 displays the suggested algorithm design, evaluation measures, and implementation techniques. The discussion and results then follow in Section 4 and 5, and the conclusion of the research is then stated in section 6 .

\section{Related work}

Before explaining our architecture in more depth, this paper's following section presents a brief introduction to the NSCT fusion strategy and deep neural network. COVID-19 or coronavirus is an updated version of pneumonia of unknown cause found in Wuhan, China, and was first confirmed in China's WHO Country Office; the disease was named COVID-19 by WHO (Cascella et al., 2020). To fight this virus's spread, the cooperation between specialists in medical and artificial intelligence is required. From this pandemic, the attempts to diagnosis, classify, detect, and specify the suitable recovery method is performed and widely spread all over the countries (Bullock et al., 2020; Shi et al., 2020; Pham et al., 2020; Elavarasan et al., 2020; Vafea et al., 2020; Raoofi et al., 2020).

Certainly, data fusion is essential to discover more details and improve observed data's extracted features (Meng et al. 2020; Attallah et al. 2020; Thabtah et al. 2020). Typically, the Generative Adversarial Network (GAN) is widely used for data augmentation, especially for small data presented by Shams et al. (Shame et al., 2020) for CXR images. In medical applications, the fusion of images is performed to discover essential parts (Tian et al., 2016). It is well known that images are in three levels: binary level 0 and one, grayscale level 0 to 255, and RGB level. Most medical images, especially CT and CXR images, are grayscale (Ran et al., 2020).

Image fusion (IF) is an essential branch of information science. IF was widely used in different fields, including medical imaging, bioinformatics, simulation of military targets, etc. (Raol et al., 2009). This paper will study the fusion strategies based NSCT, deep learning CNN-VGG19, and the hybrid of NSCT and deep learning CNN-VGG19.

\subsection{NSCT fusion strategy}

The combination of low and high-frequency coefficient of source images is called NSCT. (Bhatnagar et al., 2013) presented an architecture applied to CT and MRI images to be fused using NSCT to extract edge information and prominent texture based on directive contrast of the frequency coefficients. The main limitation is the shift variance problem that may occur in the fused images. To overcome this problem, a cascaded combination of NSCT and stationary wavelet transform (SWT) is presented by (Bhateja et al., 2015) to enhance the phase's shift variance problem information of the fused images. 
133 Precisely, pixel-level image fusion is promising in many image fusion strategies. (Li et al., 2017) 134 present multi-scale transformation coefficients to produce fused images with inter-scale 135 correlation. They apply MRI and PET images to observe fusion performance objectives and 136 determine the source images' miss-registration.

137 Hybrid decomposition of NSCT and morphological sequential toggle operator (MSTO) is 138 presented by (Wang et al., 2020). Their methodology can extract significant feature information 139 of the source images while preserving the unambiguous edges with a little produced noise in 140 both visible and infrared image fusion.

141 (Bashir et al. 2019) present an algorithm for multi-modal imagery based on SWT, and principal

142

143 component analysis (PCA) applied to CXR, CT, and MRI images.

In addition to using NSCT in fusion strategy, the noise distribution produced by CXR images can be handled using Poisson-Gaussian noise analysis, as presented by (Lee et al., 2018). The authors apply their algorithm on CXR images to reduce the resulting noise images. Moreover, (Chandra

146

147 et al. 2020) presented an algorithm to extract shape features from CXR images based on a grey level co-occurrence matrix (GLCM) with an improved abnormality detection.

2.2 Deep learning in image fusion strategiesDeep learning (DL) approaches can be used as a late step in most fusion strategies (Lee et al., 2018). Most of CT and CXR images in medical applications can be handcrafted and fused in score level fusion strategy (Baumgartl et al., 2020). Moreover, DL can be used as a feature extractor by which the fusion process is carried out in the feature extraction step. Next, the choice of features is determined using both CNN and PCA presented by Bahandary et al. (Bahandary et al., 2020). The combination of one-dimensional feature vectors and the dimensionality reduction is performed using PCA are then applied to the source CXR images and tested the normal bacterial pneumonia.

156 A proposed method based on pre-trained CNN to fuse different subsets and transfer learning classifiers is presented by (Ozkaya et al., 2020). It is applied to CT images to classify COVID-19 cases. To visualize different registration of essential data of the source images using fixed and moving data labels as well as fixed and moving images, (Haskins et al. 2020) presented an algorithm based on DCNN applied in MRI, CXR, and CT modality.

\subsection{Hybrid NSCT and CNN fusion strategies}

Hybrid techniques are generally helpful in many medical applications as it supports the strength points of approach while avoiding issues of shortage (Jaradat et al., 2009). In this study, we implement the use of a hybrid fusion technique using NSCT and CNN. The fusion of infrared and visible medical images can be performed using NSCT and dual-channel of pulse coupled neural network (PCNN) as presented by (Xiang et al., 2015). and PET scan are presented by (Lin et al., 2020). They used DSCNN and NSCT to fuse multiband images reconstructed by long short-term memory (LSTM) and DSCNN to overcome the data-driven approach's controllability problem.

Hybrid multimodality medical image fusion applied in both CXR and CT images were presented by (Rajalingam et al., 2018) based on convolutional and hybrid algorithms for disease analysis. A fusion architecture based on CNN of two source images and decomposition level based on NSCT is reconstructed using Gaussian pyramid reconstruction of the fused images (Huang et al., 2020). The maximum selection fusion rule of two source CT images based on NSCT and spatial frequency analysis of the source images are applied to the pulse coupled neural network presented as hybrid fusion architecture by (Das et al., 2012). Moreover, for MRI modality 
178 (Maharjan et al., 2020) proposed a hybrid model to detect brain tumors using NSCT and extreme 179 learning machinery (ELM).

\section{$180 \quad 2.4$ Fusion strategies in classifying COVID-19 CXR images}

181

182

183

184

185

186

187

188

189

190

191

192

193

194

195

196

197

198

199

200

201

202

203

204

205

206

207

208

209

210

211

212

213

214

215

216

217

218

219

220

221

222

223

Different approaches are recently used to classify CXR images with fusion strategies to enhance, detect, and recognize the COVID-19 cases easier and precisely. The fusion of deep learning and statistical features of the enrolled CXR images are performed to ensure the clarity of the relevant information without losing more details where the patches of CXR images of COVID-19 cases are located.

(Pereira et al., 2020) presented four phases to classify CXR images: the feature extraction, the Early Fusion technique, the data resampling, and the generation and classification of outcomes for the multi-class and hierarchical scenarios. They used both the Early and Late Fusion strategy based on recognized texture descriptors and a pre-trained CNN model. The fusion strategy is based on a weighted sum, weighted product, and the enrolled features' voting strength. They achieved an average F1-Score of 0.65 and 0.89 multi-class and hierarchical classification, respectively.

Deep feature fusion algorithm presented by (Wang et al., 2021) was utilized to fuse both individual image-level features and relation-aware features to produce Graph Convolutional Networks (GCN) and CNN, respectively. The extracted features are based on self-created CNN that learn image-level individually. The applied algorithm used to classify COVID-19 cases that assist radiologists in detecting COVID-19 cases rapidly. Commonly, deep learning fusion classifiers provide more encouraging results to detect COVID-19 cases than traditional RT-PCR testing. It made the detection and prediction process more reliable with increased accuracy (Panwar et al., 2020). They used a color visualization approach to make the deep learning model more interpretable and explainable.

Two collaborative stream networks presented by (Chen et al., 2020) are used to classify multilabel CXR images based on lung segmentation. A self-adaptive weighted fusion scheme is applied to aggregate the contextual information in both global and lung fields with the mean area under the curve $\mathrm{AUC}=0.82$. Moreover in ( $\mathrm{Li}$ et al., 2020) used multi-resolution convolutional networks to learn the features and employed four different fusion methods that are CNN, Committee, late, and Full fusion strategies for lung classification and the results obtained are $95.01 \%, 97.17 \%, 97.92 \%$, and $98.23 \%$ respectively.

Parallel-dilated convolutional neural network (PDCNN) based COVID-19 classification system from chest X-ray images is presented by (Chowdhury et al., 2020) generated features are fused into the CNN network to produce the final prediction. They used 2905 chest X-ray images representing COVID-19, Normal, and Pneumonia cases with a reasonable accuracy reached to $96.58 \%$.

COVID-19 hybrid classification approach based on a fusion of CNN and swarm-based feature selection algorithm is presented by (Sahlol et al., 2020). This combination is helpful to obtain high performance with minimum computational time. They used fractional order-marine predictors algorithm (FO-MPA) as an optimizer to select the most significant features from deep features produced from CNN that usually have redundancy; therefore, thereby depreciating the resources' capacity higher classification rate of COVID-19 X-ray images are achieved. The major limitation is eliminating the CNN redundancy, and the low quality of the fused image may produce an error in diagnosis and classification. For this reason, in diagnosis and classification issues, the need to improve the quality of the fused image is required, and it is conducive to detect the relevant features of the applied images. 
224 One of the well-known methods is Non-subsampled Contourlet Transform (NSCT). (Xinqiang et 225 al., 2020) present an image fusion method based on local neighbor features and NSCT with a 226 promising fusion effect on multi-focus images, especially medical images with infrared and 227 visible light images.

228 Therefore, in this work, we exploit the advantages of deep learning approaches with the NSCT 229 method to obtain more precise and accurate images with specific detail to diagnose CXR 230 COVID-19 cases. The best of our knowledge is the fusion of NSCT, and deep learning features 231 are not used in the CXR COVID-19 classification issue. In Table (1), we investigate the 232 summary of the related work.

Table 1: Summary of the related work

235

236

237

238

239

240

241

242

243

244

245

246

247

248

249

250

251

252

253

254

255

256

257

258

259

260

261

262

263

264

265

\section{Proposed Algorithm Framework}

The system proposed in this paper is two-layer image fusion using deep learning as shown in Figure (1). The proposed method can adaptively decompose two images or more and reconstruct the new image with a high-quality image in the fusion. Using NSCT to decompose the input images to get their high frequency and low-frequency images, and extract their features vector for each low-pass subband and high-pass subband by the CNN-VGG19, combine them our fusion method (NSCT) to achieve the final fusion images.

The system is made up of five major stages, as shown in Figure (2):

- Data preprocessing: reading CXR images Dataset in greyscale, converting to RGB, resizing, and denoising are all done in the first stage of data preprocessing.

- NSCT decomposition: image $\mathrm{X}$ and $\mathrm{Y}$ or more than 2 ready images are decomposed into their low-pass subband and high-pass subband images, respectively.

- Deep learning convolutional neural networks (VGG19): The third stage is DL using CNN-VGG19 as a feature extractor.

- Fusion rule: A temporal consistency of extracted features, Euclidean distance and weights subband calculations are used

- NSCT fusion technique: finally, the fused image is computed from the fused high-pass subband and the fused low-pass subband images by applying the inverse NSCT.

Figure 1: The Proposed algorithm framework

Figure 2: Proposed perceptual two layer image fusion using deep learning

\subsection{Materials \& Methods}

\subsubsection{Dataset Preprocessing}

The first step before the fusion model is data preprocessing, which includes the following steps:

- Initially, we start reading the datasets.

- All datasets of CXR images in greyscale are converted to RGB images to be appropriate for CNN-VGG19. 
266

267

268

269

270

271

272

273

274

275

276

277

278

279

280

281

282

283

284

285

286

287

288

289

290

291

292

293

294

295

296

297

298

299

300

301

302

303

304

305

306

- One of the significant phases in the data preprocessing is resizing the resulting RGB images. Since the dataset is collected from different waves, they have different sizes aligned into $(224,224)$.

- An aligned CXR image's appearance is enhanced using the proposed method (Dagel et al., 2008).

- Finally, the total variation for image components is denoised using the method proposed (chambolle et al., 2009).

Figure (3) indicates dataset preprocessing steps by taking an example of CXR COVID-19 image cases. The resulting histograms of distributed pixels in each step of the preprocessing reflect that the preprocessing strategy aims to maintain the pixel distribution's original essence, thus suppressing the abnormal strengths.

\section{Figure 3 (a, b, c, and d): Example of dataset preprocessing steps of raw CXR COVID-19 image}

\subsubsection{Fusion Based on NSCT}

NSCT has significant features of avoiding spectral invariance in aliasing and translation. The decomposition and reconstruction procedure preserves the source image's specifics so that the image's features can be extracted. NSCT carries out processing on the source image to obtain low-pass frequency and high-pass frequency in each direction, and by inverse NSCT a fused image is transformed as shown in Figure (4) (Huang et al., 2020).

\subsubsection{High-pass Subbands Fusion Rule}

The CXR is processed as the input image to the NSCT decomposition level stage; therefore, a fusion process integrates the trained image with enhancement performance. High-pass filter fusion's significant subbands are the augmentation process that performs each source image's specific features. Equation (1) describes the fused high-pass $\mathrm{HP}_{\mathrm{F}}$ subband image as follows.

$$
\operatorname{HP}_{F}(x, y)=\left\{\begin{array}{lr}
\operatorname{HP}_{A}(x, y) & \text { if } \operatorname{Lmap}_{A}(x, y)=1 \\
H_{B}(x, y) & \text { otherwise }
\end{array}\right.
$$

where $H P_{F}, H P_{A}$ and $H P_{B}$ are subband high-pass images for the fused image of source IA and IB images, respectively. $\operatorname{Dmap}_{A}(x, y)$ means the map decision for the high-pass sub-band as determined in Equation (2).

$$
\operatorname{Dmap}_{i}(x, y)=\left\{\begin{array}{lr}
1 & \text { if }\left[S_{i}(x, y)\right]>\frac{\tilde{Q} \times \tilde{R}}{2} \\
0 & \text { otherwise }
\end{array}\right.
$$

In Eq. 2, $S_{i}$ signifies the sliding window with a specific size of $\tilde{Q} \times \tilde{\mathrm{R}}$, and is concentrated at (x, y) with i number of source images.

\subsubsection{Low-pass Subbands Fusion Rule}

In Low-pass subbands filter, most source images' energies are contained to produce significant fused images with enhanced performance. While the NSCT filters have the most exhaustive information 
307 308 309 310 311

than the high-pass subbands, there are still restricted decomposition levels of NSCT that cannot filter all the images' information. Therefore, to ultimately preserve the detailed information of low-pass subbands, we attempt to use different measurements that reflect the fused images' structured data based on NSCT (Liu et al., 2015).

Equation (3) investigates the presence of two activity level measures that are implemented to determine the detailed information, which is the weighted sum (WS) of the 8-neighborhood, weighted local energy (WE), respectively.

WE is determined as Equation 3 as follows:

$$
W E(x, y)=\sum_{m=-r}^{r} \sum_{n=-r}^{r} \Psi \times(m+r+1, n+r+1) \times L P(x+m, y+n)^{2}
$$

where LP signifies the low-pass subband of source image at ( $\mathrm{x}, \mathrm{y})$, WE signify the localized WE at $(\mathrm{x}, \mathrm{y}), \Psi$ is a matrix that contains $(2 \mathrm{r}+1) \times(2 \mathrm{r}+1)$ and the elements in $\Psi$ are $2^{2 r-d}$. The radius of matrix $\Psi$ is $r$ and $d$ is the distance of four-neighborhood distance to the center of matrix $\Psi$. A matrix is shown in Equation (4) investigated that when $r$ is set to 1, the normalized matrix $\Psi$ is $\frac{1}{16}\left[\begin{array}{lll}1 & 2 & 1 \\ 2 & 4 & 2 \\ 1 & 2 & 1\end{array}\right]$

Here WE is utilized to measure the structured information, whereas WS is used to measure the detailed extracted features shown in Equation (5).

$\mathrm{WS}(\mathrm{x}, \mathrm{y})=\sum_{\mathrm{m}=-\mathrm{r}}^{\mathrm{r}} \sum_{\mathrm{n}=-\mathrm{r}}^{\mathrm{r}} \Psi(\mathrm{m}+\mathrm{r}+1, \mathrm{n}+\mathrm{r}+1) \times \xi(\mathrm{x}+\mathrm{m}, \mathrm{y}+\mathrm{n})$

$\Psi$ is the weighted matrix investigated in Equation (3), while the $\xi$ is illustrated in Equation (6). The parameter $\xi$ indicated that full usage of the neighbored information was performed. Therefore, exhaustive information can be restored by $\xi$. In this scenario, When $\xi$ and WS are achieved, the fusion of both low-pass subband images can be determined by the rule proposed in Equation (7), given that $L P_{F}, L P_{A}$, and $L P_{B}$ are low-pass subband images of the fused image given the source image $I_{A}$ and $I_{B}$ respectively. $\mathrm{WL}_{A}^{N}$ and $\mathrm{WS}_{B}^{N}$ are the normalized WS of $I_{A}$ and $I_{B}$ respectively.

$$
\begin{aligned}
& \xi(x, y)=|2 L(x, y)-L(x-1, y)-L(x+1, y)| \\
& \quad+|2 L(x, y)-L(x, 1, y)-L(x+1, y)| \\
& \quad+\frac{1}{\sqrt{2}}|2 L(x, y)-L(x-1, y-1)-L(x+1, y+1)| \\
& \quad+\frac{1}{\sqrt{2}}|2 L(x, y)-L(x-1, y+1)-L(x+1, y-1)|
\end{aligned}
$$


$\operatorname{LP}_{F}(x, y)=\left\{\begin{array}{l}\operatorname{LP}_{A}(x, y) \\ \operatorname{LP}_{B}(x, y)\end{array}\right.$

$$
\begin{aligned}
& \text { if } 0.5 \cdot \operatorname{WLE}_{\mathrm{A}}^{\mathrm{N}}(\mathrm{x}, \mathrm{y})+0.5 \\
& \cdot \mathrm{WS}_{\mathrm{A}}^{\mathrm{N}}(\mathrm{x}, \mathrm{y}) \geq 0.5 \cdot \mathrm{WLE}_{\mathrm{B}}^{\mathrm{N}}(\mathrm{x}, \mathrm{y}) \\
& +0.5 \cdot \mathrm{WS}_{\mathrm{B}}^{\mathrm{N}}(\mathrm{x}, \mathrm{y})
\end{aligned}
$$

346

347

348

349

350

351

352

353

354

355

356

357

358

359

360

361

362

363

364

365

366

367

368

369

370

371

372

373

374

375

376

377

378

379

380

381

382

383

384

385

The fused coefficients of high-pass subband $\left(\mathrm{H}_{\mathrm{F}}\right)$ and low-pass subband $\left(\mathrm{L}_{\mathrm{F}}\right)$ are determined, the resulting fused image $\left(\mathrm{I}_{\mathrm{F}}\right)$ can be acquired by inverting NSCT over $\left\{H_{F}, L_{F}\right\}$. The inverse transformation of the NSCT is realized by optimizing linear reconstruction for $\mathrm{H}_{\mathrm{F}}$ and $\mathrm{L}_{\mathrm{F}}$ based on dual coordinate system operators.

Finally, to obtain a fused image, the image is reverse by NSCT. In Figure (4), a block diagram of the NSCT-based fusion approach is shown.

\section{Figure 4: The NSCT fusion method}

\subsubsection{Convolutional Neural Network Architectures}

Recently, the usage in all-purpose of DL algorithms and CNNs has directed several innovations in a diversity of computerized applications, such as object segmentation, classification, and recognition (LeCun et al., 2015). DL methods have proven effective in automating learning to represent features and characteristics while actively seeking to remove handcrafted features engineering's repetitive task. By adding a hierarchical layer of feature representation, the DL and CNNs aim to mimic the human visual cortex system's purpose and construction.

After the 2012 ImageNet competition, CNNs have been commonly used in image processing problems. In a convolution layer, the output feature map is produced when the preceding layer's feature maps are converted to learnable kernels by using the activation function. Multiple input maps will combine convolutions with each output map. It is generally formulated as it is in Equation 8.

$x_{j}^{l}=f\left(\sum_{i \in M_{j}} x_{i}^{l-1} * k_{i j}^{l}+b_{j}^{l}\right)$

Within Equation 8, $\mathrm{M}_{\mathrm{j}}$ denotes the range of an input map. If both output map $\mathrm{j}$ and map $\mathrm{k}$ both sum over input map $i$, then the kernels added to map $i$ are distinct (Liu et al., 2015), for output maps $\mathrm{j}$ and $\mathrm{k}$.

\subsubsection{Convolutional Neural Networks for Features Extraction}

\section{Spatial Exploitation based on CNN's}

CNN's consist of a relatively large number of hyperparameters and parameters, such as neurons, number of layers, biases, filter sizes, stride, learning rate, activation function, and weights. Here, different correlation levels can be explored based on other filters as the combinatorial process considers the local area of the input pixels. Different filter sizes encapsulate various complexity levels; small filters typically extract fine-grained while large filters extract coarse-grained data. 
386

387

388

389

390

391

392

393

394

395

396

397

398

399

400

401

402

403

404

405

406

407

408

409

410

411

412

413

414

415

416

417

418

419

420

421

422

423

424

425

426

427

428

429

430

431

As a result, researchers exploited spatial filters in early 2000 to enhance quality and explored a spatial filter relationship with network learning. Multiple kinds of research were published in this era indicated that $\mathrm{CNN}$ could perform effectively on coarse and fine-grained data when modifying filters.

The technical descriptions of different CNN models, their parameters and principal contribution, rate of error, categorization, and depth are summarized in Table (2) (Khan et al.2020)

\section{Table 2: Performance comparison of the recent CNN models}

\subsubsection{VGGNet}

The experiment in structural technology has accelerated with the active use of CNNs in image classification tasks. A simple and efficient design theory for CNN architectures was proposed by Simonyan et al. in this regard. Their style, known as VGG, was modular in layer structure patterns compared to AlexNet and ZfNet; VGG was rendered 19 layers deep to visualize the depth with the network's truly representative capability (Simonyan et al., 2014). ZfNet, the 2013-ILSVRC competition frontline network, indicated that limited filtering could increase the efficiency of CNNs. Based on the results obtained, VGG displaced the $11 \times 11$ and $5 \times 5$ filters with a $3 \times 3$ filter layer stack and demonstrated experimentally that the concomitant positioning of small filters ( $3 \times 3)$ could produce the impact of large filter sizes $(5 \times 5$ and $7 \times 7)$. By decreasing the number of variables, small filters offer an additional advantage of low computational complexity. These results set a new trend in research for $\mathrm{CNN}$ to work with narrower filters. By putting 1x1 convolutions between the convolutional layers, VGG controls a network's configuration, learning a feature vector of the resulting feature maps. For both image classification and localization challenges, VGG provided excellent performance. In the 2014-ILSVRC competition, VGG had been in second place, and due to its simple design, heterogeneous configuration, and improved scale, it gained popularity. The critical drawback associated with VGG is that the use of 138 million variables, making it costly and challenging to implement computationally on lowresource systems (Khan et al., 2020). The graphical representation of VGG19 adopted from (Özyurt et al., 2019) is investigated in Figure (5), Table (3).

\section{Figure 5: Blocks graphical representation of VGG19}

\section{Table 3: Characteristics of VGG19 used in the proposed framework}

We used a standard and effective CNN model in this work, named VGGNet, illustrated in Figure (5), with 16 convolutional and three layers of wholly connected. The convolutional layers' width is comparably small, rising by a factor between 64 in the initial layer to 512, of 2 during each process of max-pooling. There is a reasonable size of 224 x 224 pixels on the input layer. A stride is implemented to retain spatial resolution since each image is transferred through a convolution stack. Pooling is done throughout a fixed window by five max-pooling layers with stride following some but not all convolutional layers. During the first two, three completely connected layers with 4096 channels are accompanied by a stack of convolution layers with depth varying in various configurations, during the third complete identification.

Temporal consistency is an effective methodology for capturing the contrasting harmony in any input sequence image, especially gray-level images. In turn, for any gray level change in the 
432 input sequence, even though at least one must be in the fused sequence without any change in 433 contrast or delay. When CXR image sequences are combined, there is a problem reflected in the 434 merging sequence images' consistency because light stimuli' movement has a responsive effect 435 on the human visual system (Rockinger et al., 1998). The contrast changes introduced by the 436 fusion process will therefore be very distracting, and therefore we will apply (El-Gamal et al., 437 2016).

438 A simplified pseudo-code implementation of two-layer image fusion using deep learning is

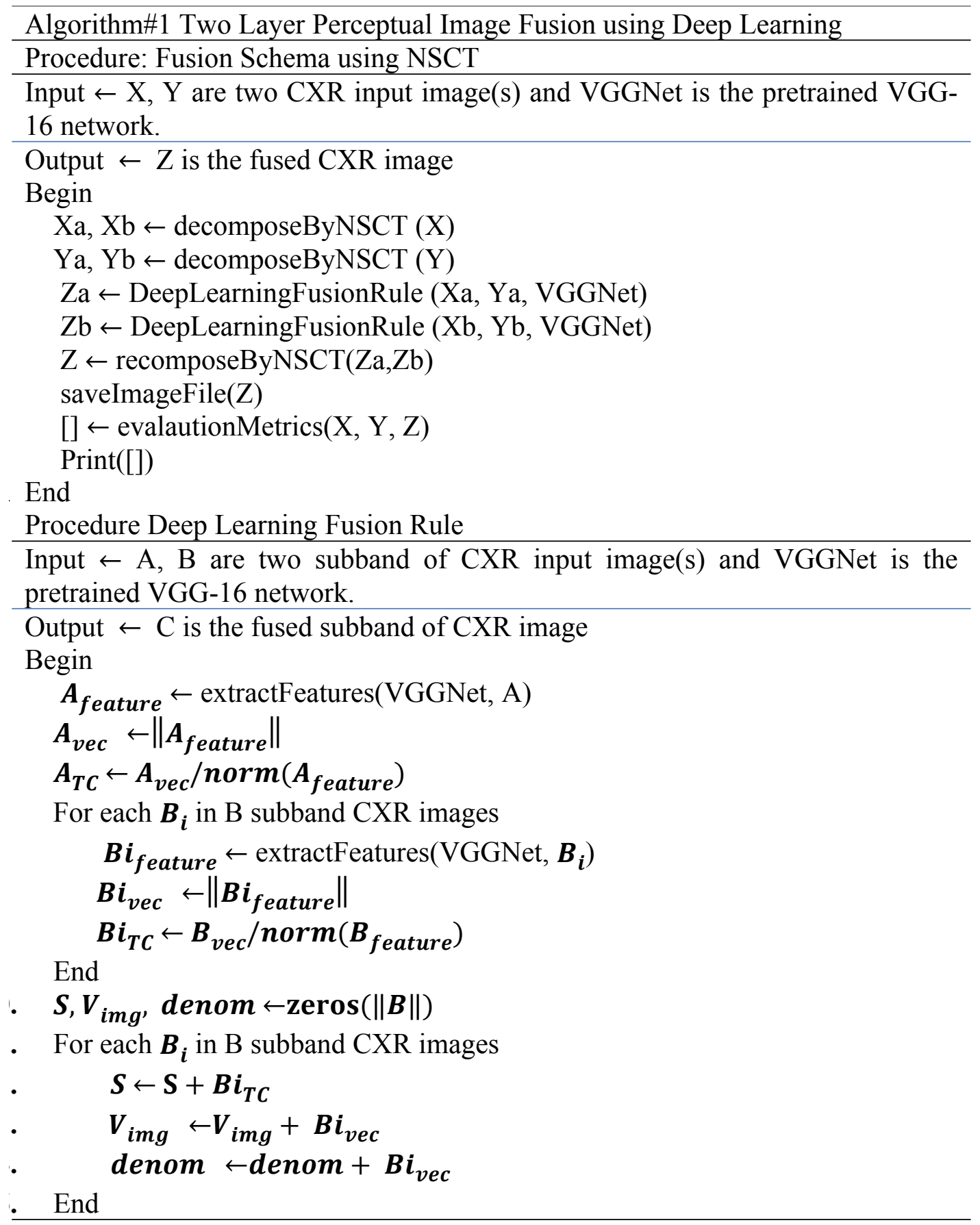


- For each $\boldsymbol{B}_{\boldsymbol{i}}$ in B subband CXR images

- $\quad W \leftarrow B i_{T C} /$ denom

- End

- $\quad C \leftarrow \operatorname{zeros}(\|B\|)$

- For each $\boldsymbol{W}_{\boldsymbol{i}}$ in $\mathrm{W}$

$\boldsymbol{C} \leftarrow \boldsymbol{C}+\boldsymbol{B}_{\boldsymbol{i}} * \boldsymbol{W}_{\boldsymbol{i}}$

- End

. End

441

442

\section{Evaluation Matrices}

443 This section is dedicated to exploring the effectiveness of the proposed approach. Two different 444 experimental studies were carried out, discussed, and analyzed in detail due to the variability of 445 the updated standard datasets versions of X-ray COVID-19 images.

446 All experiments were carried out using the MATLAB 2019b software package running on 447 Microsoft machine with Core i7 processor, 16-RAM, and NVIDIA 4G-GT 740m GPU 448 environment. This section presents a dataset description, validation, and the findings of adding a 449 convolutional deep neural network to NSCT fusion method.

450

451

452

453

454

455

456

457

458

459

460

461

462

463

464

465

466

467

468

469

470

471

472

473

\subsection{COVID-19 Dataset and Evaluation Metrics}

\subsubsection{COVID-19 Dataset}

The Dataset used for this work includes 87 chest X-ray images acquired on 25 cases (17 male, 7 females, and 1 blank) all of which were confirmed with COVID-19. The CXR COVID-19 images cases are available at the Kaggle repository, CXR COVID-19 Dataset (Cohen et al., 2020), existing at https://www.kaggle.com/bachrr/covid-chest-xray.

In this study, a clinical dataset for CXR COVID-19 images was utilized for training and validation. This Dataset consists of images for 25 patients, it has 2 images or more for each patient. Figure (6) shows four samples CXR images from Dataset for 1 patient (Bachrr Kaggle, 2020). Table (4) investigated the complete datasets, including patient data and class labels, and can be demonstrated as follows.

Table 4. The collected Dataset that describes different features related to each patient

Figure 6. Sample of Dataset

(CXR COVID-19 images of a 53-year-old patient with pneumonia after 10 days of infection)

\subsubsection{Evaluation Metrics}

Performance Analysis needs to be evaluated using a consistently approved standard of image fusion quality. $\mathrm{Q}^{\mathrm{MI}}$ (Quality Mutual Information), Standard Deviation (STD), Peak Signal to Noise Ratio (PSNR), Structural Similarity Index Measure (SSIM), $\mathrm{Q}^{\mathrm{AB} / \mathrm{Fmetric}}$, and Spatial Frequency (SF) are the evaluation measures used and were applied as follows (Xydeas et al., 2000; Yang et al., 2008; Hossny et al., 2008; Chen et al., 2009; and Chen, et al., 2011;). 


\section{4.1.2.1 Standard Deviation (STD)}

475 To measure the global divergence of the fused image, the standard deviation is practically used.

476 Furthermore, the difference between the data obtained and the mean is calculated using it. More

477 useful information from the fused image is obtained when the STD value is higher, as investigated in

478 Equation (9).

$479 \quad \sigma^{2}=\sqrt{\frac{\sum_{i=1}^{I} \Sigma_{j=1}^{J}(f(i, j)-\mu)^{2}}{M N}}$

480 where (I) and (J) are the length and width of the fused image $f(i, j)$, and is generally determined as

481256 with the mean value of the merged image $(\mu)$.

482

483

\subsubsection{Quality Mutual Information $\left(Q^{\mathrm{MI}}\right)$}

484 Generally, MI is the degree of dependence amongst 2 source images (X, Y). MI investigated the 485 amount of calculated information that represents the source image concerning the fused image. The 486 MI denoted by $(\mathcal{M})$ is relative to the fused message by which the formula $(\mathcal{M})$ can be defined as 487 Equations (10-14):

488

$489 \mathcal{M}=I(x, f)+I(y, f)$,

$490 \quad I(X, Y)=\sum_{y \in Y} \sum_{x \in Y} p(x, y) \log \frac{p(x, y)}{p(x) p(y)}$

491

492

493

494 495

496

497

498

499

500

$501 \mathrm{I}(\mathrm{X}, \mathrm{X})=\mathrm{H}(\mathrm{X})$

502 Hence:

$503 \quad \mathrm{Q}^{\mathrm{MI}}=2\left[\frac{\mathrm{I}(\mathrm{F}, \mathrm{X})}{\mathrm{H}(\mathrm{F})+\mathrm{H}(\mathrm{X})}+\frac{\mathrm{I}(\mathrm{F}, \mathrm{Y})}{\mathrm{H}(\mathrm{F})+\mathrm{H}(\mathrm{Y})}\right]$

504

505

506 image. as in Equation (12): since is realized as in Equation (13):

where $p(x)$ and $p(y)$ represents the Probability Density Functions (PDF) of the two images, and $p(x$, y) represents the Joint-Probability Density Function (JPDF) of the source image X, Y, and fused

To estimate the dependability between the random variables $\mathrm{X}$, and $\mathrm{Y}$, the $\mathrm{I}(\mathrm{X}, \mathrm{Y})$ can be determined

$\mathrm{I}(\mathrm{X}, \mathrm{Y})=\sum_{\mathrm{y} \in \mathrm{Y}} \sum_{\mathrm{x} \in \mathrm{Y}} \mathrm{p}(\mathrm{x}, \mathrm{y}) \log \frac{\mathrm{p}(\mathrm{x}, \mathrm{y})}{\mathrm{p}(\mathrm{x}) \mathrm{p}(\mathrm{y})}=0$

Given that $\mathrm{X}, \mathrm{Y}$ and $\mathrm{F}$ are the histogram normalization of the source images $\mathrm{x}, \mathrm{y}$ with the resulting fused image $\mathrm{f}$, respectively. By applying MI, the problems regarding the boundness of the metric

where $\mathrm{H}(\mathrm{X}), \mathrm{H}(\mathrm{Y})$ and $\mathrm{H}(\mathrm{F})$ represents the entropies of $\mathrm{X}, \mathrm{Y}$ and $\mathrm{F}$, respectively.

\subsubsection{Peak Signal to Noise Ratio (PSNR)}


507 It is well-known that PSNR is a quantitative indicator depending on Mean Square Error (MSE). The 508 large value of PSNR leads to improve the fused image and enhancement of SNR of the source image 509 Equation (15).

$510 \quad$ PSNR $=10 \times \log _{10}\left(\frac{\mathrm{L}^{2}}{\mathrm{RMSE}^{2}}\right)$

511 Given the PSNR denotes the maximum grey pixel value of the fused image, which is 255 . The

512 RMSE can be determined as in Equation (16) by which it represents the difference between the

513 source images and the fused images.

514

$\operatorname{RMSE}=\sqrt{\frac{\sum_{\mathrm{m}=1}^{\mathrm{M}} \sum_{\mathrm{n}=1}^{\mathrm{N}}[\operatorname{ground}(\mathrm{m}, \mathrm{n})-\operatorname{fused}(\mathrm{m}, \mathrm{n})]^{2}}{\mathrm{M} \times \mathrm{N}}}$

515 RMSE reflects the fused image's ability compared with Ground $(m, n)$ to determine the error with the applied length and width of the image with size $\mathrm{M}$ and $\mathrm{N}$, respectively.

\section{4.1.2.4 Structural Similarity Index Measure (SSIM)}

518 One of the essential benchmarks for the similarity evaluation of the fused and source images are

519 SSIM by which the structural similarity (SSIM) metric of the corresponding regions is determined as 520 in Equation (17) as follows:

$521 \operatorname{SSIM}(x, y \mid w)=\frac{\left(2 \bar{w}_{x} \overline{\mathrm{w}}_{\mathrm{y}}+\mathrm{C}_{1}\right)\left(2 \sigma_{w_{x} w_{y}}+\mathrm{C}_{2}\right)}{\left(\bar{w}_{x}^{2}+\bar{w}_{y}^{2}+C_{1}\right)\left(\sigma_{w_{x}}^{2}+\sigma_{w_{y}}^{2}+C_{2}\right)}$

522 Which can be decomposed as

$523 \operatorname{SSIM}(x, y \mid w)=\frac{\left(2 \bar{w}_{\mathrm{x}} \overline{\mathrm{w}}_{\mathrm{y}}+\mathrm{C}_{1}\right)\left(2 \sigma_{w_{x} w_{y}}+\mathrm{C}_{2}\right)\left(\sigma_{w_{x} w_{y}}+\mathrm{C}_{3}\right)}{\left(\bar{w}_{\mathrm{x}}^{2}+\overline{\mathrm{w}}_{\mathrm{y}}{ }^{2}+C_{1}\right)\left(\sigma_{w_{x}}^{2}+\sigma_{w_{y}}^{2}+C_{2}\right)\left(\sigma_{w_{x}} \sigma_{w_{y}}+\mathrm{C}_{3}\right)}$

524 Given that the parameters $\mathrm{C}_{1}, \mathrm{C}_{2}$ and $\mathrm{C}_{3}$ represent the small constants such that $\mathrm{C}_{3}=\mathrm{C}_{2} / 2$, and the $\mathrm{w}_{\mathrm{x}}$

525 denotes the sliding region in $\mathrm{x}$, so that $\bar{w}_{\mathrm{x}}$ is the mean of $\mathrm{x}, \sigma_{w_{x}}^{2}$ and $\sigma_{w_{x}{ }_{y}}$ represents both variance 526 and covariance of the $\mathrm{x}$ and $\mathrm{y}$, respectively.

\section{4.1.2.5 Spatial Frequency (SF)}

528 SF determines the sharpness of the image in fusion. Besides, SF is calculated as the change rate in the 529 grey level of the image. As in Equation (19), the greater the SF, the higher the image quality.

$530 S F=\sqrt{R F^{2}+C F^{2}}$

531 Moreover, 
532

533

534

535

536

537

538

539

540

541

542

543

544

545

546

547

548

549

550

551

552

553

554

555

556

557

558

559

560

561

562

563

564

$$
\begin{aligned}
& R F=\sqrt{\frac{1}{M(N-1)}} \sum_{i=1}^{M} \sum_{j=2}^{N}(X(i, j-1)-X(i, j))^{2} \\
& C F=\sqrt{\frac{1}{(M-1) N}} \sum_{i=2}^{M} \sum_{j=1}^{N}(X(i, j)-X(i-1, j))^{2}
\end{aligned}
$$

where RF and CF represent the row and column frequencies of the image, respectively.

\subsubsection{6 $Q^{\mathrm{AB} / \mathrm{F}}$ Measurement}

We used the $\mathrm{Q}^{\mathrm{AB} / \mathrm{F}}$ parameter determined by the Sobel edge detection operator to evaluate the amount of edge information in the fused image compared to the source images. The higher value of $\mathrm{Q}^{\mathrm{AB} / \mathrm{F}}$ denotes, the extra data is renewed from the source image, and the edge information is improved and conserved. Generally, the great edge strength produce a great impact on $\mathrm{Q}^{\mathrm{AB} / \mathrm{F}}$ as in Equation (22)

$$
Q^{A B / F}=\frac{\sum_{n=1}^{N} \sum_{m=1}^{M}\left(Q^{A}(n, m) W^{A}(n, m)+Q^{B}(n, m) W^{B}(n, m)\right)}{\sum_{n=1}^{N} \sum_{m=1}^{M}\left(W^{A}(i, j)+W^{B}(i, j)\right)}
$$

where $\mathrm{Q}^{\mathrm{A}}(\mathrm{n}, \mathrm{m}), \mathrm{Q}^{\mathrm{B}}(\mathrm{n}, \mathrm{m})$ is the edge information storage value; $W^{A}(n, m), W^{B}(n, m)$ is the weighting map.

\section{Experimental Results and Discussion}

\subsection{Statistical Analysis}

In this paper, we determine the mentioned parameters statistically in the previous section. These parameters include the average, standard deviation, min, max, and median of the fused features obtained in the training phase. Different standard image fusion quality performance metrics including $\mathrm{Q}^{\mathrm{MI}}, \mathrm{STD}, \mathrm{PSNR}, \mathrm{SSIM}, \mathrm{Q}^{\mathrm{AB} / \mathrm{F}}$, and SF are used for evaluation and analysis a statistical study which was applied as follows (Chen et al., 2011; Hossny et al., 2008; Xydeas et al., 2000; Yang et al., 2008; Chen et al., 2009).

\section{Table 5: Statistical analysis of the fused CXR COVID-19 images}

Table (5) indicates the performance metric values in terms of $\mathrm{Q}^{\mathrm{AB} / \mathrm{F}}, \mathrm{Q}^{\mathrm{MI}}, \mathrm{PSNR}, \mathrm{SSIM}$, SF, and STD. Various experiments are performed independently for different traits of the fused CXR COVID-19 images. From this Table (5), the following remarks could be concluded as follows;

- $\quad$ Higher values of PSNR are produced at the maximum level (Max value is 20.91); consequently, the (average value is 16.92).

- The Standard Deviation (STD) is determined in both sample STDEV.S and population STDEV.P, and the result of the fused CXR COVID-19 images are in between 0.02 to 0.23 maximum value.

- $\quad$ These results indicate that the proposed fusion strategy is stabled during the training process as the statistical balancing in the obtained results are achieved.

\subsection{Performance comparison of the recent $\mathrm{CNN}$ architectures of different categories}

Six well-known convolutional neural networks were used to study the effect of feature extraction and extraction time: AlexNet, VGG-16, VGG-19, GoogleNet, ResNet-50, and ResNet-101. The best 
565

566

567

568

569

570

571

572

573

574

575

576

577

578

579

580

581

582

583

584

585

586

587

588

589

590

591

592

593

594

595

596

597

598

599

600

601

602

603

604

performance was achieved among all CNN networks by VGG-19, as shown in Figure (7). VGG19 has the smallest value, 512 feature vector length, with a minimum extraction time of $0.543489 \mathrm{sec}$. Therefore, VGG19 is used in the proposed framework to satisfy the trade-off between feature vector length and extraction time.

\section{Figure 7 (a, and b): Comparative results of different $\mathrm{CNN}$ and the proposed framework} CNN-VGG19

\subsection{Evaluation Performance of CXR COVID-19 Images for Image Fusion using DL}

\subsubsection{CXR Imaging Characteristics}

CXR entering the entity can be sensed in the CXR imaging process, such as Compton scattering effect and other effects to create CXR attenuation, as the thickness or density of the object to be measured varies, the energy attenuation is also diverse. Thus, screening or film creates a CXR image with black and white contrast, with a gradual increase of the CXRs' dose in penetrating objects, and an increase in the image region's brightness. Once the tube voltage is constant, the tube current increases, the number of photons in the scan range increases, and the entire CXR image's greyscale increases (Chen et al., 2011).

Figures (8), and (9) show images and histogram distribution for CXR COVID-19 images input and fused CXR COVID-19 images with a diverse radiation source dose. In the image's background region, when the grey scale difference is small, then the histogram of this image appears at a single peak and the grey scale near 1 as shown in figure (8-c) or the grey scale near zero as shown in figure (9-c). Whereas in the fused CXR COVID-19 images, the grey scale value is high in the overall image, and the histogram background region is spread over the greyscale as shown in figures (8-d), and (9-d). In our patients' results, the CXR COVID-19 images had high contrast and many features.

\section{Figure 8: Example of a female 50-year-old patient $\left(P_{1}\right)$ CXR COVID-19 images fusion} Figure 9: Example of a male 67-year-old patient $\left(P_{6}\right)$ CXR COVID-19 images fusion

Table (6) includes the fused CXR COVID-19 image. The value is denoted in bold lettering, which presents the evaluation parameters of the different datasets of the various random patients $\left(\mathrm{P}_{1}, \mathrm{P}_{2}\right.$, .., and $\mathrm{P}_{7}$ ) of input and fused CXR COVID-19 images. Two metrics are used for measurable performance evaluations, such as SF and STD, to assess the proposed algorithm performance. The larger values of the SF for the fused image in all patients indicates the higher image resolution. For fused CXR COVID-19 images, the highest STD value is calculated. Then without distortion, the proposed algorithm can maintain the CXR COVID-19 image, the fused image is more apparent than input images, and the effect of fusion is powerful.

Table 6: Evaluation indicator data of various patients (Source (Input image X, Input image Y) and fused CXR COVID-19 images)

Figure 10: Comparison between input image $X$ and fused image of evaluation indicator SF Figure 11: Comparison between input image $Y$ and fused image of evaluation indicator SF 
605

606

607

608

609

610

611

612

613

614

615

616

617

618

619

620

621

622

623

624

625

626

627

628

629

630

631

632

633

634

635

636

637

638

639

640

In Figure (10), the proposed CXR COVID-19 image fused algorithm produces CXR COVID-19 images for random seven patients during seven experiments using only two CXR COVID-19 images. Our proposal generates a CXR COVID-19 image with SF greater than or equal to SF of the input image(s). When there are more than two CXR COVID-19 images, we compromise the highest and lowest resolution image to guarantee our proposal's best performance.

Figure (11) shows that the generated CXR COVID-19 image gains a significant SF value against the first input CXR COVID-19 image values. Since the SF measures the sharpness of objects in the CXR COVID-19 image and grey level change ratio, the proposed fusion method leads to better judgment and diagnosis of the patient from his CXR COVID-19 image(s).

\section{Figure 12: Comparison between input image $X, Y$ and fused image of evaluation indicator STD}

A comparative evaluation is performed for the two input CXR COVID-19 image and the produced CXR COVID-19 image based on the STD evaluation metric during seven experiments for random seven patients. The proposed algorithm generates significant STD values five times higher than the two input images versus the same STD values as shown in Figure (12). We can compromise that the proposed fusion method is efficient enough to generate CXR COVID-19 images that are more useful for the examiner for exploring patient status.

\subsection{Comparative Analysis}

\subsubsection{Validation Performance using Evaluation Metrics}

The algorithms for evaluation performance, by comparison, are GFF (Li et al., 2013), MSA (Du et al., 2016), NSCT+LE (Zhu et al., 2019), NSST+PAPCNN (Yin et al., 2018), respectively. Six metrics are practical to objective evaluation metrics measurements, such as $\mathrm{Q}^{\mathrm{AB} / \mathrm{F}}, \mathrm{Q}^{\mathrm{MI}}, \mathrm{PSNR}$, SSIM, SF, and STD (Du et al., 2016), to determine the competence of the various multi-modal MIF algorithms mentioned above.

In Table (7), The highest $\mathrm{Q}^{\mathrm{AB} / \mathrm{F}}$ value is the GFF algorithm. The result is that the GFF algorithm protects the input image's edge information. The GFF algorithm also achieves good results on the evaluation metric for the SF. This algorithm enhances the resolution of the fused image by utilizing guided filtering to display each pixel's saliency and spatial accuracy. The algorithm NSST+PAPCNN performs best on the indicator STD of evaluation, leading to purer fused images.

The proposed algorithm NSCT+CNN_VGG19 also accomplishes good results on the Q ${ }^{\mathrm{MI}}$, SSIM, and PSNR evaluation metric measures. The use of DL to extract the features improves the proposed algorithm's performance, so the NSCT + CNN-VGG19 algorithm achieves the highest in 
641 the $\mathrm{Q}^{\mathrm{MI}}$, PSNR, SSIM. The higher PSNR value means the pixel grey value is higher than the 642 comparative algorithms in the fused image. Also, the great benefit of SSIM means that the fused 643 image and the input image are structurally identical to other algorithms. The higher values of $644 \mathrm{Q}^{\mathrm{MI}}$, PSNR, SSIM, as shown in Table (7) and Figure (13), mean that the fusion effects of images 645 are strong compared to competitive approaches.

646

647

648

649

650

651

652

653

654

655

656

657

658

659

660

661

662

663

664

665

666

667

668

669

670

671

672

673

674

675

676

677

678

679

Table 7: Fused images evaluation metrics of different algorithms

\section{Figure 13 (a, and b): Representation of evaluation index data of different methods of fused images}

This study compared the evaluation metrics of the different proposals. Upon prior knowledge of the authors, $\mathrm{Q}^{\mathrm{AB} / \mathrm{F}}, \mathrm{Q}^{\mathrm{MI}}$, PSNR, SSIM, SF, and STD were deemed significant metrics in evaluating the fusion method. In turn, the achieved points of the proposal versus other research was compared as shown in Figure (14) and found that:

1) Two proposals (MSA (Du et al., 2016) and NSCT+LE (Zhu et al., 2019)) did not meet any of the evaluation measures.

2) Yin, $M$. et al. scored a total of $33 \%$ of the overall evaluation metrics $\left(Q^{A B / F}\right.$ and $\left.S F\right)$ to evaluate the performance of the algorithm (NSST+PAPCNN (Yin et al., 2018)).

3) The proposed research achieved $50 \%$ of the overall evaluation metrics in $\mathrm{Q}^{\mathrm{MI}}$, PSNR, SSIM

Figure 14: Cluster Dendrogram Hierarchy Evaluation of the proposed algorithm with competitive metrics of fused methods for images

\subsubsection{Validation Performance using classification}

To assess the performance of the proposed framework, we used accuracy, sensitivity, and specificity. The descriptions of these measures are as follows:

Accuracy $=\frac{\mathrm{TP}+\mathrm{TN}}{\mathrm{TP}+\mathrm{TN}+\mathrm{FP}+\mathrm{FN}}$

(23)

Sensitivity $=\frac{\mathrm{TP}}{\mathrm{TP}+\mathrm{TN}}$

Specificity $=\frac{\mathrm{TN}}{\mathrm{TN}+\mathrm{TP}}$

Where:

'TP': true positives cases in an instance of COVID-19 case, 'TN': true negatives cases, significantly negative COVID-19 case, 'FP': false positives cases, and 'FN': false negatives cases are the incorrect categorized by the classifier for COVID-19.

The evaluation of fused CXR COVID-19 images for the pre-trained proposed framework image fusion using DL classification was performed for the COVID-19 dataset using deep learning and compared accuracy in recognizing the different systems as shown in Table (8). The dataset that is already used in classification comparison is the same dataset used in (Abbas et al., 2020; Apostolopoulos et al., 2020; and Luz et al., 2020).

Peer) Comput. Sci. reviewing PDF | (CS-2020:10:53728:2:1:NEW 29 Dec 2020) 
680

681

682

683

684

685

686

687

688

689

690

691

692

693

694

695

696

697

698

699

700

701

702

703

704

705

706

707

708

709

710

711

712

713

714

715

716

717

718

719

720

721

722

\section{Table 8: Comparison with other CNN architectures}

Decompose, transfer, and compose (DeTraC) model for detecting COVID-19 from CXR images was presented (Abbas et al., 2020). They used different pre-trained transfer learning model based on both shallow and deep learning approaches. They tested their method with and without decomposition, and the VGG-19 accuracy is determined in both shallow and deep learning tuning mode, and the resulting accuracy was $93.42 \%$ and $94.59 \%$, respectively.

(Apostolopoulos et al., 2020) presented a transfer learning approach to detect and classify COVID-19 cases from normal cases automatically. They used VGG-19 compared with other transfer learning methods, and the achieved results based on accuracy, sensitivity, and specificity were $98.75 \%, 92.85 \%$, and $98.75 \%$, respectively.

(Luz et al., 2020) presented an architecture based on CNN to detect the abnormality caused by COVID-19 using 13569, 231 trained, and tested cases, respectively. They tested the three-class labels normal, pneumonia, and COVID-19 using the well-known transfer learning approaches VGG-19, VGG-16, and Resnet-15, and the resulting accuracy was $75.3 \%, 77.0 \%$, and $83.5 \%$, respectively.

As present in Table (8), The pre-trained proposed model using Shallow (KNN) compared with other pre-trained models and achieved results based on accuracy, sensitivity, and specificity were $96.93 \%, 57.14 \%$, and $99.2 \%$, respectively. The pre-trained proposed model using Deep Learning (CNN-VGG19) compared with other pre-trained models and achieved results based on accuracy, sensitivity, and specificity was $99.04 \%, 85.71 \%$, and $99.6 \%$, respectively. In turn, classification rates (accuracy and specificity) indicating the result of fusion as enhancement and riching image with extra details as a side effect of classification in CXR vision application.

Due to the insights in terms of average precision, average recall, and accuracy that is mined based on Figure (15), the proposed fusion method helps either shallow classifier, KNN, and deep learning classifier (CNN-VGG19) in the classification task. In turn, the fusion is a positive initiative for enhancing the classification and aided computer vision models using CXR COVID19 images.

\section{Figure 15 (a, and b): (a) Confusion matrix of the pre-trained proposed framework using VGG19 for CXR COVID-19 images and (b) Confusion matrix of the pre-trained proposed framework using KNN for CXR COVID-19 images}

\section{Conclusion}

MIF growth varies from the spatial domain, transformation domain, to DL. Its rapid growth also suggests a strong demand for computer-aided clinical diagnosis. Different researchers suggest various fusion methods, each of which has its advantages in the multiple measures of evaluation indicators. However, there exist approximately thirty types of evaluation indicators for MIF. Furthermore, the fusion techniques developed depend on early methods. The researchers enhanced the existing problems in fusion but did not resolve it; distortion of colors and features extraction. In MIF, innovative algorithms remain a major challenge in this field of research.

Peer] Comput. Sci. reviewing PDF | (CS-2020:10:53728:2:1:NEW 29 Dec 2020) 
723 DL has strengthened the effect of fusion, but research also has some rules; DL structure, for 724 instance, is single, and the amount of training data is limited. Because professional labelling by 725 medical experts is required for the qualified CXR images, they hardly work, and the cost is high. 726 Therefore, there is also a lack of data for training, eventually leading to overfitting.

727 A fast and effective diagnostic test or protocol will help achieve appropriate early medical care 728 for COVID-19 patients, helping save many lives worldwide. Finding a rapid and effective 729 diagnostic protocol or test becomes one of the critical priorities. This paper is one step ahead 730 towards implementing Deep Learning-based fusion methods to obtain more informative CXR 731 images for the COVID-19 dataset. It could aid in screening or accelerate the speed of COVID73219 diagnosis. We observe that in the COVID-19 CXR image's background region, when the 733 greyscale difference is small, then the histogram of this image appears at a single peak and the 734 greyscale near one as shown in Figure (8-c) or the greyscale near zero as shown in Figure (9-c). 735 Whereas in the fused CXR COVID-19 images, the greyscale value is high in the overall image, 736 and the histogram background region is spread over the greyscale as shown in Figures (8-d) and 737 (9-d). All the fused images show the same features, and it works in both hard and light cases. 738 The fused algorithm proposed works well in all cases, and its appearance in the Evaluation 739 indicator data of various random patients, as shown in Tables 6 as an example.

740 The future trend is the study of DL in MIF, according to the previous section. This research 741 proposes a novel MIF algorithm based on DL for Imbalanced COVID-19 Dataset 742 (NSCT+CNN_VGG19). Thus, hybrid decomposition and fusion of NSCT and CNN_VGG19 as 743 features extractor is also used. The proposed algorithm can determine that the proposed fusion 744 method is efficient enough to generate CXR COVID-19 images that are more useful for the 745 examiner for exploring patient status. The comparative evaluation was performed using two 746 methods; the first method is to determine the pre-trained framework efficiency using evaluation 747 metrics. While the second method is based on classifying the fused CXR COVID-19 images 748 using the deep learning approach CNN-VGG19 compared with the state-of-the-art.

749 A comparison was also undergone to evaluate the different MFI algorithms performance models

750 using six metrics as evaluation measures; these are $\mathrm{Q}^{\mathrm{AB} / \mathrm{F}}, \mathrm{Q}^{\mathrm{MI}}$, PSNR, SSIM, SF, and STD. The 751 proposed algorithm NSCT + CNN_VGG19 performed best on the predictor of Q ${ }^{\mathrm{MI}}$, PSNR, SSIM 752 evaluation measures. The image was more apparent, and the fused image contained extensive 753 information. Finally, the suggested algorithm is more efficient than comparable approaches.

754 The results demonstrate that the pre-trained proposed framework using fusion based on NSCT

755 with deep learning VGG19 may significantly affect the classification and feature extraction from 756 X-ray COVID-19 images automatically related to the diagnosis COVID-19.

757

\section{Compliance with Ethical Standards}

759 On behalf of all authors, the corresponding author states that there is no conflict of interest. This 760 work neither contains any study on animals nor humans performed by any of the authors. All 761 authors contributed equally to the work. 


\section{References}

764 Ganasala, P., \& Kumar, V. (2016). Feature-motivated simplified adaptive PCNN-based medical

Srivastava, R., Prakash, O., \& Khare, A. (2016). Local energy-based multimodal medical image fusion in curvelet domain. IET computer vision, 10(6), 513-527.

Zhu, Z., Yin, H., Chai, Y., Li, Y., \& Qi, G. (2018). A novel multi-modality image fusion method based on image decomposition and sparse representation. Information Sciences, 432, 516-529.

Zhu, Z., Zheng, M., Qi, G., Wang, D., \& Xiang, Y. (2019). A phase congruency and local Laplacian energy based multi-modality medical image fusion method in NSCT domain. IEEE Access, 7, 20811-20824.

Liu, S., Shi, M., Zhu, Z., \& Zhao, J. (2017). Image fusion based on complex-shearlet domain with guided filtering. Multidimensional Systems and Signal Processing, 28(1), 207-224.

Liu, H., Wang, L., Nan, Y., Jin, F., Wang, Q., \& Pu, J. (2019). SDFN: Segmentation-based deep fusion network for thoracic disease classification in chest X-ray images. Computerized Medical Imaging and Graphics, 75, 66-73.

Huang, Z., Lin, J., Xu, L., Wang, H., Bai, T., Pang, Y., \& Meen, T. H. (2020). Fusion HighResolution Network for Diagnosing ChestX-ray Images. Electronics, 9(1), 190.

Hu, M., Lin, H., Fan, Z., Gao, W., Yang, L., Liu, C., \& Song, Q. (2020). Learning to Recognize Chest-Xray Images Faster and More Efficiently Based on Multi-Kernel Depthwise Convolution. IEEE Access, 8, 37265-37274.

Tian, Yu, Yibing Li, and Fang Ye. "Multi-modal medical image fusion based on nonsubsampled contourlet transform using improved PCNN." 2016 IEEE 13th International Conference on Signal Processing (ICSP). IEEE, 2016.

Gomathi, Periyavattam Shanmugam, and Bhuvanesh Kalaavathi. "Multi-modal medical image fusion in non-subsampled contourlet transform domain." Circuits and Systems 7.8 (2016): 1598-1610.

Bhatnagar, Gaurav, QM Jonathan Wu, and Zheng Liu. "Directive contrast based multi-modal medical image fusion in NSCT domain." IEEE transactions on multimedia 15.5 (2013): 1014-1024.

Liu, Y., Chen, X., Wang, Z., Wang, Z. J., Ward, R. K., \& Wang, X. (2018). Deep learning for pixel-level image fusion: Recent advances and future prospects. Information Fusion, 42, 158-173.

Liu, Y., Chen, X., Peng, H., \& Wang, Z. (2017). Multi-focus image fusion with a deep convolutional neural network. Information Fusion, 36, 191-207.

Lahoud, F., \& Süsstrunk, S. (2019). Fast and efficient zero-learning image fusion. arXiv preprint arXiv:1905.03590.

Hermessi, H., Mourali, O., \& Zagrouba, E. (2018). Convolutional neural network-based multimodal image fusion via similarity learning in the shearlet domain. Neural Computing and Applications, 30(7), 2029-2045.

Amin-Naji, M., Aghagolzadeh, A., \& Ezoji, M. (2019). Ensemble of CNN for multi-focus image fusion. Information fusion, 51, 201-214.

Tang, H., Xiao, B., Li, W., \& Wang, G. (2018). Pixel convolutional neural network for multifocus image fusion. Information Sciences, 433, 125-141. 
807 Cascella, M., Rajnik, M., Cuomo, A., Dulebohn, S. C., \& Di Napoli, R. (2020). Features, 808 evaluation and treatment coronavirus (COVID-19). In Statpearls [internet]. StatPearls

809

810

811

812

813

814

815

816

817

818

819

820

821

822

823

824

825

826

827

828

829

830

831

832

833

834

835

836

837

838

839

840

841

842

843

844

845

846

847

848

849

850

851

Publishing.

Bullock, J., Pham, K. H., Lam, C. S. N., \& Luengo-Oroz, M. (2020). Mapping the landscape of artificial intelligence applications against COVID-19. arXiv preprint arXiv:2003.11336.

Shi, F., Wang, J., Shi, J., Wu, Z., Wang, Q., Tang, Z., ... \& Shen, D. (2020). Review of artificial intelligence techniques in imaging data acquisition, segmentation and diagnosis for covid-19. IEEE reviews in biomedical engineering.

Pham, Q. V., Nguyen, D. C., Hwang, W. J., \& Pathirana, P. N. (2020). Artificial Intelligence (AI) and Big Data for Coronavirus (COVID-19) Pandemic: A Survey on the State-ofthe-Arts.

Elavarasan, R. M., \& Pugazhendhi, R. (2020). Restructured society and environment: A review on potential technological strategies to control the COVID-19 pandemic. Science of The Total Environment, 138858.

Vafea, M. T., Atalla, E., Georgakas, J., Shehadeh, F., Mylona, E. K., Kalligeros, M., \& Mylonakis, E. (2020). Emerging Technologies for Use in the Study, Diagnosis, and Treatment of Patients with COVID-19. Cellular and Molecular Bioengineering, 1-9.

Raoofi, A., Takian, A., Sari, A. A., Olyaeemanesh, A., Haghighi, H., \& Aarabi, M. (2020). COVID-19 pandemic and comparative health policy learning in Iran. Archives of Iranian Medicine, 23(4), 220-234.

Meng, T., Jing, X., Yan, Z., \& Pedrycz, W. (2020). A survey on machine learning for data fusion. Information Fusion, 57, 115-129.

Attallah, O., Sharkas, M. A., \& Gadelkarim, H. (2020). Deep Learning Techniques for Automatic Detection of Embryonic Neurodevelopmental Disorders. Diagnostics, 10(1), 27.

Shams M.Y., Elzeki O.M., Abd Elfattah M., Medhat T., Hassanien A.E. (2020) Why Are Generative Adversarial Networks Vital for Deep Neural Networks? A Case Study on COVID-19 Chest X-Ray Images. Big Data Analytics and Artificial Intelligence Against COVID-19: Innovation Vision and Approach. Studies in Big Data, vol 78. Springer, Cham. 147-162.

Thabtah, F., \& Peebles, D. (2020). A new machine learning model based on induction of rules for autism detection. Health informatics journal, 26(1), 264-286.

Ran, A., Chen, S., Zhang, S., Liu, S., Zhou, Z., Nie, P., ... \& Kang, F. (2020). A gradient screening approach for retired lithium-ion batteries based on CXR computed tomography images. RSC Advances, 10(32), 19117-19123.

Raol, J. R. (2009). Multi-sensor data fusion with MATLAB. CRC press.

Bhatnagar, G., Wu, Q. J., \& Liu, Z. (2013). Directive contrast based multi-modal medical image fusion in NSCT domain. IEEE transactions on multimedia, 15(5), 1014-1024.

Bhateja, V., Patel, H., Krishn, A., Sahu, A., \& Lay-Ekuakille, A. (2015). Multi-modal medical image sensor fusion framework using cascade of wavelet and contourlet transform domains. IEEE Sensors Journal, 15(12), 6783-6790.

Li, S., Kang, X., Fang, L., Hu, J., \& Yin, H. (2017). Pixel-level image fusion: A survey of the state of the art. information Fusion, 33, 100-112.

Kraetschmer, T., Dagel, D., \& Sanders, S. T. (2008). Simple multiwavelength time-division multiplexed light source for sensing applications. Optics letters, 33(7), 738-740.

Peer) Comput. Sci. reviewing PDF | (CS-2020:10:53728:2:1:NEW 29 Dec 2020) 
852 Chambolle, A., Caselles, V., Cremers, D., Novaga, M., \& Pock, T. (2010). An introduction to

853

854

855

856

857

858

859

860

861

862

863

864

865

866

867

868

869

870

871

872

873

874

875

876

877

878

879

880

881

882

883

884

885

886

887

888

889

890

891

892

893

894

895

896 total variation for image analysis. Theoretical foundations and numerical methods for sparse recovery, $9(263-340), 227$

Wang, Z., Xu, J., Jiang, X., \& Yan, X. (2020). Infrared and visible image fusion via hybrid decomposition of NSCT and morphological sequential toggle operator. Optik, 201, 163497.

Bashir, R., Junejo, R., Qadri, N. N., Fleury, M., \& Qadri, M. Y. (2019). SWT and PCA image fusion methods for multi-modal imagery. Multimedia Tools and Applications, 78(2), 1235-1263.

Lee, S., Lee, M. S., \& Kang, M. G. (2018). Poisson-Gaussian noise analysis and estimation for low-dose CXR images in the NSCT domain. Sensors, 18(4), 1019.

Chandra, T. B., Verma, K., Singh, B. K., Jain, D., \& Netam, S. S. (2020). Automatic Detection of Tuberculosis Related Abnormalities in CXR Images using Hierarchical Feature Extraction Scheme. Expert Systems with Applications, 113514.

Lee, Sheng Long, Mohammad Reza Zare, and Henning Muller. "Late fusion of deep learning and handcrafted visual features for biomedical image modality classification." IET image processing 13.2 (2018): 382-391.

Baumgartl, H., Tomas, J., Buettner, R., \& Merkel, M. (2020). A deep learning-based model for defect detection in laser-powder bed fusion using in-situ thermographic monitoring. Progress in Additive Manufacturing, 1-9.

Bhandary, A., Prabhu, G. A., Rajinikanth, V., Thanaraj, K. P., Satapathy, S. C., Robbins, D. E., ... \& Raja, N. S. M. (2020). Deep-learning framework to detect lung abnormality-A study with CXR and lung CT scan images. Pattern Recognition Letters, 129, 271-278.

Ozkaya, U., Ozturk, S., \& Barstugan, M. (2020). Coronavirus (COVID-19) Classification using Deep Features Fusion and Ranking Technique. arXiv preprint arXiv:2004.03698.

Haskins, G., Kruger, U., \& Yan, P. (2020). Deep learning in medical image registration: a survey. Machine Vision and Applications, 31(1), 8.

Jaradat, M. A. K., \& Langari, R. (2009). A hybrid intelligent system for fault detection and sensor fusion. Applied Soft Computing, 9(1), 415-422.

Xiang, T., Yan, L., \& Gao, R. (2015). A fusion algorithm for infrared and visible images based on adaptive dual-channel unit-linking PCNN in NSCT domain. Infrared Physics \& Technology, 69, 53-61.

Lin, Suzhen, Ze Han, Dawei Li, Jianchao Zeng, Xiaoli Yang, Xinwen Liu, and Feng Liu. "Integrating model-and data-driven methods for synchronous adaptive multiband image fusion." Information Fusion 54 (2020): 145-160.

Rajalingam, B., Priya, R., \& Bhavani, R. (2018). Comparative analysis for various traditional and hybrid multi-modal medical image fusion techniques for clinical treatment analysis. Image Segmentation: A Guide to Image Mining, ICSES Transactions on Image Processing and Pattern Recognition (ITIPPR). ICSES Publisher, 26-50.

Das, S., \& Kundu, M. K. (2012). NSCT-based multi-modal medical image fusion using pulsecoupled neural network and modified spatial frequency. Medical \& biological engineering \& computing, 50(10), 1105-1114.

Maharjan, S., Alsadoon, A., Prasad, P. W. C., Al-Dalain, T., \& Alsadoon, O. H. (2020). A novel enhanced softmax loss function for brain tumour detection using deep learning. Journal of Neuroscience Methods, 330, 108520. 
897 Pereira, Rodolfo M., Diego Bertolini, Lucas O. Teixeira, Carlos N. Silla Jr, and Yandre MG

898

899

900

901

902

903

904

905

906

907

908

909

910

911

912

913

914

915

916

917

918

919

920

921

922

923

924

925

926

927

928

929

930

931

932

933

934

935

936

937

938

939

940

941

942 Costa. "COVID-19 identification in chest X-ray images on flat and hierarchical classification scenarios." Computer Methods and Programs in Biomedicine (2020): 105532.

Wang, Shui-Hua, Vishnu Varthanan Govindaraj, Juan Manuel Górriz, Xin Zhang, and Yu-Dong Zhang. "Covid-19 classification by FGCNet with deep feature fusion from graph convolutional network and convolutional neural network." Information Fusion 67 (2020): 208-229.

Panwar, Harsh, P. K. Gupta, Mohammad Khubeb Siddiqui, Ruben Morales-Menendez, Prakhar Bhardwaj, and Vaishnavi Singh. "A deep learning and grad-CAM based color visualization approach for fast detection of COVID-19 cases using chest X-ray and CTScan images." Chaos, Solitons \& Fractals 140 (2020): 110190.

Chen, Bingzhi, Zheng Zhang, Jianyong Lin, Yi Chen, and Guangming Lu. "Two-Stream Collaborative Network for Multi-label Chest X-ray Image Classification with Lung Segmentation." Pattern Recognition Letters (2020).

Li, Xuechen, Linlin Shen, Xinpeng Xie, Shiyun Huang, Zhien Xie, Xian Hong, and Juan Yu. "Multi-resolution convolutional networks for chest X-ray radiograph-based lung nodule detection." Artificial intelligence in medicine 103 (2020): 101744.

Chowdhury, Nihad K., Md Muhtadir Rahman, and Muhammad Ashad Kabir. "PDCOVIDNet: a parallel-dilated convolutional neural network architecture for detecting COVID-19 from chest X-ray images." Health information science and systems 8, no. 1 (2020): 1-14.

Sahlol, Ahmed T., Dalia Yousri, Ahmed A. Ewees, Mohammed AA Al-Qaness, Robertas Damasevicius, and Mohamed Abd Elaziz. "COVID-19 image classification using deep features and fractional-order marine predators' algorithm." Scientific Reports 10, no. 1 (2020): 1-15.

Xinqiang, Qin, Zheng Jiaoyue, and $\mathrm{Hu}$ Gang. "Image fusion method based on the local neighborhood feature and nonsubsampled contourlet transform." In 2017 2nd International Conference on Image, Vision and Computing (ICIVC), pp. 396-400. IEEE, 2017.

Huang, B., Yang, F., Yin, M., Mo, X., \& Zhong, C. (2020). A Review of Multimodal Medical Image Fusion Techniques. Computational and Mathematical Methods in Medicine, 2020.

Liu, Y., Liu, S., \& Wang, Z. (2015). A general framework for image fusion based on multi-scale transform and sparse representation. Information fusion, 24, 147-164.

LeCun, Y., Bengio, Y., \& Hinton, G. (2015). Deep learning. nature, 521(7553), 436-444.

Canziani, A., Paszke, A., \& Culurciello, E. (2016). An analysis of deep neural network models for practical applications. arXiv preprint arXiv:1605.07678.

Simonyan, K., \& Zisserman, A. (2014). Very deep convolutional networks for large-scale image recognition. arXiv preprint arXiv:1409.1556.

Khan, A., Sohail, A., Zahoora, U., \& Qureshi, A. S. (2020). A survey of the recent architectures of deep convolutional neural networks. Artificial Intelligence Review, 53(8), 5455-5516.

Krizhevsky, A., Sutskever, I., \& Hinton, G. E. (2017). Imagenet classification with deep convolutional neural networks. Communications of the ACM, 60(6), 84-90.

He, K., Zhang, X., Ren, S., \& Sun, J. (2016). Deep residual learning for image recognition. In Proceedings of the IEEE conference on computer vision and pattern recognition (pp. 770-778). 
943 Szegedy, C., Vanhoucke, V., Ioffe, S., Shlens, J., \& Wojna, Z. (2016). Rethinking the inception

944

945

946

947

948

949

950

951

952

953

954

955

956

957

958

959

960

961

962

963

964

965

966

967

968

969

970

971

972

973

974

975

976

977

978

979

980

981

982

983

984

985

986

987 architecture for computer vision. In Proceedings of the IEEE conference on computer vision and pattern recognition (pp. 2818-2826).

Özyurt, F. (2019). Efficient deep feature selection for remote sensing image recognition with fused deep learning architectures. The Journal of Supercomputing, 1-19.

Rockinger, O., \& Fechner, T. (1998, July). Pixel-level image fusion: the case of image sequences. In Signal processing, sensor fusion, and target recognition VII (Vol. 3374, pp. 378-388). International Society for Optics and Photonics.

El-Gamal, F. E. Z. A., Elmogy, M., \& Atwan, A. (2016). Current trends in medical image registration and fusion. Egyptian Informatics Journal, 17(1), 99-124.

https://www.kaggle.com/bachrr/covid-chest-xray Accessed August 2020.

Chen, F., Pan, J., \& Han, Y. (2011). An effective image quality evaluation method of CXR imaging system. Journal of Computational Information Systems, 7(4), 1278-1285.

Hossny, M., Nahavandi, S., \& Creighton, D. (2008). Comments on 'Information measure for performance of image fusion'. Electronics letters, 44(18), 1066-1067.

Xydeas, C. A., \& Petrovic, V. (2000). Objective image fusion performance measure. Electronics letters, 36(4), 308-309.

Yang, C., Zhang, J. Q., Wang, X. R., \& Liu, X. (2008). A novel similarity-based quality metric for image fusion. Information Fusion, 9(2), 156-160.

Chen, Y., \& Blum, R. S. (2009). A new automated quality assessment algorithm for image fusion. Image and vision computing, 27(10), 1421-1432.

Chen, F., Pan, J., \& Han, Y. (2011). An effective image quality evaluation method of CXR imaging system. Journal of Computational Information Systems, 7(4), 1278-1285.

Li, S., Kang, X., \& Hu, J. (2013). Image fusion with guided filtering. IEEE Transactions on Image processing, 22(7), 2864-2875.

Du, J., Li, W., Xiao, B., \& Nawaz, Q. (2016). Medical image fusion by combining parallel features on multi-scale local extrema scheme. Knowledge-Based Systems, 113, 4-12.

Zhu, Z., Zheng, M., Qi, G., Wang, D., \& Xiang, Y. (2019). A phase congruency and local Laplacian energy based multi-modality medical image fusion method in NSCT domain. IEEE Access, 7, 20811-20824.

Yin, M., Liu, X., Liu, Y., \& Chen, X. (2018). Medical image fusion with parameter-adaptive pulse coupled neural network in nonsubsampled shearlet transform domain. IEEE Transactions on Instrumentation and Measurement, 68(1), 49-64.

Du, J., Li, W., Lu, K., \& Xiao, B. (2016). An overview of multi-modal medical image fusion. Neurocomputing, 215, 3-20.

Abbas, A., Abdelsamea, M.M. \& Gaber, M.M. Classification of COVID-19 in chest X-ray images using DeTraC deep convolutional neural network. Appl Intell (2020). https://doi.org/10.1007/s10489-020-01829-7.

Apostolopoulos, Ioannis D., and Tzani A. Mpesiana. "Covid-19: automatic detection from x-ray images utilizing transfer learning with convolutional neural networks." Physical and Engineering Sciences in Medicine (2020): 1.

Luz, Eduardo José da S., Pedro Lopes Silva, Rodrigo Silva, Ludmila Silva, Gladston Moreira, and David Menotti. "Towards an Effective and Efficient Deep Learning Model for COVID-19 Patterns Detection in X-ray Images." CoRR (2020). 


\section{Table $\mathbf{1}$ (on next page)}

Summary of the related work 


\begin{tabular}{|c|c|c|c|}
\hline Author & Modality & Methodology & Discussion \\
\hline $\begin{array}{l}\text { Wang et al., } \\
2021\end{array}$ & $\begin{array}{l}\text { COVID-19 } \\
\text { Images }\end{array}$ & $\mathrm{GCN}+\mathrm{CNN}$ & $\begin{array}{l}\text { The fusion of both individual image- } \\
\text { level features and relation-aware } \\
\text { features to produce Graph } \\
\text { Convolutional Networks (GCN), and } \\
\text { CNN respectively }\end{array}$ \\
\hline $\begin{array}{l}\text { Wang et al. } \\
2020\end{array}$ & kurtosis map & $\begin{array}{l}\text { Hybrid decomposition of NSCT } \\
\text { and morphological sequential } \\
\text { toggle operator (MSTO) }\end{array}$ & $\begin{array}{l}\text { Their methodology extracted major } \\
\text { feature information of the source } \\
\text { images and preserved the unambiguous } \\
\text { edges with a little produced noise in } \\
\text { both visible and infrared image fusion. }\end{array}$ \\
\hline $\begin{array}{l}\text { Chandra et al. } \\
2020\end{array}$ & CXR images & $\begin{array}{l}\text { grey level co-occurrence matrix } \\
\text { (GLCM) }\end{array}$ & $\begin{array}{l}\text { extract shape feature from CXR images } \\
\text { based on grey level co-occurrence } \\
\text { matrix (GLCM) with an improved } \\
\text { abnormality detection. }\end{array}$ \\
\hline $\begin{array}{l}\text { Bahandary et } \\
\text { al. } 2020\end{array}$ & CXR images & $\mathrm{CNN}$ and PCA & $\begin{array}{l}\text { The combination of one-dimensional } \\
\text { feature vectors and the dimensionality } \\
\text { reduction is performed using PCA are } \\
\text { then applied to the source CXR images } \\
\text { and tested for the normal bacterial } \\
\text { pneumonia. }\end{array}$ \\
\hline $\begin{array}{l}\text { Ozakaya et al. } \\
2020\end{array}$ & CT images & DCNN & $\begin{array}{l}\text { pre-trained CNN to fuse different } \\
\text { subsets and transfer learning classifiers } \\
\text { to classify COVID-19 cases }\end{array}$ \\
\hline $\begin{array}{c}\text { Haskins et al. } \\
2020\end{array}$ & $\begin{array}{l}\text { MR, CXR, and } \\
\text { CT modality. }\end{array}$ & DCNN & $\begin{array}{l}\text { To visualize different registration of } \\
\text { essential data of the source images } \\
\text { using fixed and moving data labels as } \\
\text { well as fixed, and moving images }\end{array}$ \\
\hline Lin et al. 2020 & $\begin{array}{l}\text { CT, MR, and } \\
\text { PET scan }\end{array}$ & $\begin{array}{l}\text { stacked convolution neural } \\
\text { network (DSCNN) for multi } \\
\text { band images }\end{array}$ & $\begin{array}{l}\text { DSCNN and NSCT fuse multiband } \\
\text { images reconstructed by long short- } \\
\text { term memory (LSTM) and DSCNN to } \\
\text { overcome the data-driven approach's } \\
\text { controllability problem. }\end{array}$ \\
\hline $\begin{array}{l}\text { Huang et al. } \\
2020\end{array}$ & MRI-CT & NSCT and DCNN & $\begin{array}{l}\text { (NSCT) by which the fusion technique } \\
\text { able to avoid the spectral aliasing and } \\
\text { provide more characteristic of the } \\
\text { invariance translation }\end{array}$ \\
\hline $\begin{array}{l}\text { Maharjan et al. } \\
\qquad 2020\end{array}$ & Brain Tumor CT & ELM and NSCT & $\begin{array}{l}\text { detect brain tumor using NSCT and } \\
\text { extreme learning machinery (ELM]. }\end{array}$ \\
\hline $\begin{array}{l}\text { Pereira et al., } \\
2020\end{array}$ & $\begin{array}{l}\text { CXR images } \\
\text { COVID-19 }\end{array}$ & $\begin{array}{c}\text { multi-class } \\
\text { hierarchical CNN }\end{array}$ & $\begin{array}{l}\text { The fusion strategy based on weighted } \\
\text { sum, weighted product, and the voting } \\
\text { strength of the enrolled features. They } \\
\text { achieved average F1-Score of } 0.65 \text {, and } \\
0.89 \text { multi-class, and hierarchical } \\
\text { classification respectively. }\end{array}$ \\
\hline $\begin{array}{c}\text { Panwar et al., } \\
2020\end{array}$ & $\begin{array}{l}\text { Chest X-ray and } \\
\text { CT-Scan images }\end{array}$ & $\begin{array}{l}\text { deep learning and grad-CAM } \\
\text { based color visualization }\end{array}$ & $\begin{array}{l}\text { Color visualization approach to make } \\
\text { the deep learning model more }\end{array}$ \\
\hline
\end{tabular}




\begin{tabular}{|c|c|c|c|}
\hline & $\begin{array}{l}\text { of COVID-19 } \\
\text { cases }\end{array}$ & & interpreTable and explainable. \\
\hline $\begin{array}{l}\text { Chen et al., } \\
2020\end{array}$ & $\begin{array}{l}\text { Multi-label CXR } \\
\text { image }\end{array}$ & $\begin{array}{c}\text { self-adaptive } \\
\text { weighted fusion scheme }\end{array}$ & $\begin{array}{l}\text { contextual information in both global } \\
\text { and lung field with the mean area under } \\
\text { the curve AUC }=0.82 \text {. }\end{array}$ \\
\hline $\begin{array}{l}\text { Chowdhury et } \\
\text { al., } 2020\end{array}$ & $\begin{array}{c}2905 \text { chest X-ray } \\
\text { images } \\
\text { COVID-19 }\end{array}$ & $\mathrm{PDCNN}+\mathrm{CNN}$ & $\begin{array}{l}\text { Accuracy }=96.58 \text { for COVID-19, } \\
\text { Normal, and Pneumonia cases. }\end{array}$ \\
\hline $\begin{array}{l}\text { Bashir et al. } \\
2019\end{array}$ & $\begin{array}{l}\mathrm{CXR}, \mathrm{CT} \text {, and } \\
\mathrm{MRI}\end{array}$ & $\begin{array}{l}\text { multimodal imagery based on } \\
\text { using SWT and principal } \\
\text { component analysis (PCA) }\end{array}$ & $\begin{array}{l}\text { A dimensionality reduction is } \\
\text { performed using PCA and then SWT to } \\
\text { extract features }\end{array}$ \\
\hline Lee et al. 2018 & CXR images & $\begin{array}{l}\text { Poisson-Gaussian noise } \\
\text { analysis }\end{array}$ & $\begin{array}{l}\text { NSCT in fusion strategy, the noise } \\
\text { distribution produced by CXR images }\end{array}$ \\
\hline $\begin{array}{l}\text { Rajalingam et } \\
\text { al. } 2018\end{array}$ & CXR and CT & NSCT and DCNN & $\begin{array}{l}\text { Hybrid multimodality medical image } \\
\text { fusion are applied in both CXR and CT } \\
\text { images. }\end{array}$ \\
\hline Li et al. 2017 & $\begin{array}{l}\text { MRI and PET } \\
\text { images }\end{array}$ & $\begin{array}{l}\text { Multi-scale transformation } \\
\text { coefficients to produce a fused } \\
\text { image with inter-scale } \\
\text { correlation. }\end{array}$ & $\begin{array}{l}\text { They apply MRI and PET images to } \\
\text { observe the objectives of fusion } \\
\text { performance and determine the source } \\
\text { images' miss-registration. }\end{array}$ \\
\hline
\end{tabular}




\section{Table 2 (on next page)}

Characteristics of CCN used in the Proposed Framework 
Table 2: Performance comparison of the recent CNN models

\begin{tabular}{|c|c|c|c|c|c|c|c|}
\hline $\begin{array}{c}\text { CNN } \\
\text { models }\end{array}$ & Year & $\begin{array}{c}\text { Principal } \\
\text { contribution }\end{array}$ & Parameters & $\begin{array}{c}\text { Rate of } \\
\text { error }\end{array}$ & Depth & Categorization & Reference \\
\hline VGGNet & 2014 & $\begin{array}{l}\text { - Homogeneous } \\
\text { topology } \\
\text { - Using fewer } \\
\text { filters }\end{array}$ & $138 \mathrm{M}$ & $\begin{array}{l}\text { ImageN } \\
\text { et: } 7.3\end{array}$ & 19 & $\begin{array}{c}\text { Spatial } \\
\text { Exploitation }\end{array}$ & $\begin{array}{l}\text { (K. } \\
\text { Simonyan, } \\
\text { et al. 2014) }\end{array}$ \\
\hline AlexNet & 2012 & $\begin{array}{l}\text { - More deep } \\
\text { and broader than } \\
\text { the LeNet } \\
\text { - Using Relu, } \\
\text { drop and overlap } \\
\text { Pooling } \\
\text { - NVIDIA GTX } \\
580 \text { GPU }\end{array}$ & $60 \mathrm{M}$ & $\begin{array}{l}\text { ImageN } \\
\text { et: } 16.4\end{array}$ & 8 & $\begin{array}{c}\text { Spatial } \\
\text { Exploitation }\end{array}$ & $\begin{array}{l}\text { (A. Kr } \\
\text { izhevsky } \\
\text { et al. } \\
\text { 2012) }\end{array}$ \\
\hline ResNet & 2016 & $\begin{array}{l}\text { - Residual } \\
\text { training } \\
\text { - Identity object } \\
\text { tracking skip } \\
\text { connection }\end{array}$ & $\begin{array}{c}25.6 \mathrm{M} \\
1.7 \mathrm{M}\end{array}$ & $\begin{array}{l}\text { ImageN } \\
\text { et: } 3.6 \\
\text { CIFAR- } \\
10: 6.43\end{array}$ & $\begin{array}{l}152 \\
110\end{array}$ & $\begin{array}{l}\text { Depth and } \\
\text { Multipath }\end{array}$ & $\begin{array}{l}\text { (K. He et } \\
\text { al. 2015) }\end{array}$ \\
\hline $\begin{array}{c}\text { GoogleN } \\
\text { et }\end{array}$ & 2015 & $\begin{array}{l}\text { - Introduced } \\
\text { principle of } \\
\text { block } \\
\text { - Divide the } \\
\text { idea of } \\
\text { transformation } \\
\text { and fusion }\end{array}$ & $4 \mathrm{M}$ & $\begin{array}{l}\text { ImageN } \\
\text { et: } 6.7\end{array}$ & 22 & $\begin{array}{c}\text { Spatial } \\
\text { Exploitation }\end{array}$ & $\begin{array}{l}(\mathrm{C} . \\
\text { Szegedy et } \\
\text { al. 2015) }\end{array}$ \\
\hline
\end{tabular}




\section{Table 3 (on next page)}

The collected dataset that describe different features related to each patient 
Table 3: Characteristics of VGG19 used in the proposed framework

\begin{tabular}{|c|c|c|c|}
\hline Layer & Patch Size/Stride & Depth & Output Size \\
\hline Convolution & $3 \times 3 \times 64 / 1$ & 2 & $224 \times 224 \times 64$ \\
\hline Max pool & $3 \times 3 / 2$ & 1 & $112 \times 112 \times 64$ \\
\hline Convolution & $3 \times 3 \times 128 / 1$ & 2 & $112 \times 112 \times 128$ \\
\hline Max pool & $3 \times 3 / 2$ & 1 & $56 \times 56 \times 128$ \\
\hline Convolution & $3 \times 3 \times 256 / 1$ & 3 & $56 \times 56 \times 256$ \\
\hline Max pool & $1 \times 1 \times 256 / 1$ & 1 & $28 \times 28 \times 256$ \\
\hline Convolution & $3 \times 3 / 2$ & 3 & $28 \times 28 \times 512$ \\
\hline Max pool & $3 \times 3 \times 512 / 1$ & 1 & $14 \times 14 \times 512$ \\
\hline Convolution & $1 \times 1 \times 512 / 1$ & 1 & $14 \times 14 \times 512$ \\
\hline Max pool & $3 \times 3 / 2$ & 3 & $7 \times 7 \times 512$ \\
\hline Fully connected & $1 \times 3 \times 512 / 1$ & 1 & $1 \times 4096$ \\
\hline Softmax & $3 \times 3 / 2$ & 1 & $1 \times 1000$ \\
\hline
\end{tabular}




\section{Table 4 (on next page)}

Evaluation indicator data of various patients (source and fused CXR Covid_19 images) 
1

2

\begin{tabular}{|c|c|}
\hline Class Labels & Patient Data \\
\hline Patient ID & Internal Patient Identifier \\
\hline Offset & $\begin{array}{l}\text { Is very useful to provide as there are several images for the same patient to track } \\
\text { progression while being imaged, the number of days after the beginning of symptoms } \\
\text { for each image. }\end{array}$ \\
\hline Gender & Blank, Male, or Female. \\
\hline Age & Patient age in Years \\
\hline Result & Pneumonia? \\
\hline Survival & Have they survived? Yes or No \\
\hline Sight & $\mathrm{PA}, \mathrm{AP}$, or L for CXRs \\
\hline Modality & CT, CXR, or something else \\
\hline Date & Date of the acquisition of the image \\
\hline Position & Relevance from right to left (hospital name, area, state, country). \\
\hline Medical Notes & In specific, about the radiograph, not objective patient. \\
\hline
\end{tabular}

Table 4: The collected dataset describes different features related to each patient

\section{Patient Data}

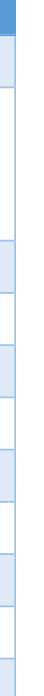




\section{Table 5 (on next page)}

Fused images evaluation metrics of different algorithms 
Table 5: Statistical analysis of the fused CXR COVID-19 images

\begin{tabular}{|c|c|c|c|c|c|c|}
\hline \multirow{2}{*}{ Statistical Measure } & \multicolumn{7}{|c|}{ Performance Metrics } \\
\hline & $\mathbf{Q}^{\mathbf{A B} / \mathbf{F}}$ & $\mathbf{Q}^{\mathbf{M I}}$ & $\mathbf{P S N R}$ & $\mathbf{S S I M}$ & $\mathbf{S F}$ & STD \\
\hline Average & 0.54 & 0.56 & 16.92 & 0.80 & 0.68 & 0.21 \\
\hline STDEV.S & 0.05 & 0.07 & 2.48 & 0.03 & 0.20 & 0.02 \\
\hline STDEV.P & 0.05 & 0.06 & 2.29 & 0.03 & 0.18 & 0.02 \\
\hline Min & 0.49 & 0.48 & 13.78 & 0.76 & 0.30 & 0.18 \\
\hline Max & 0.61 & 0.65 & 20.91 & 0.84 & 0.95 & 0.23 \\
\hline Median & 0.53 & 0.58 & 17.75 & 0.81 & 0.68 & 0.21 \\
\hline
\end{tabular}

2 


\section{Table 6(on next page)}

Evaluation indicator data of various random patients 
1

2

3

\begin{tabular}{|c|c|c|c|c|c|c|c|c|}
\hline \multirow{2}{*}{\multicolumn{2}{|c|}{$\begin{array}{l}\text { Evaluation } \\
\text { Parameters }\end{array}$}} & \multicolumn{7}{|c|}{ Patients } \\
\hline & & $\mathbf{P}_{1}$ & $\mathbf{P}_{2}$ & $\mathbf{P}_{3}$ & $\mathbf{P}_{4}$ & $\mathbf{P}_{5}$ & $\mathbf{P}_{6}$ & $\mathbf{P}_{7}$ \\
\hline \multirow{3}{*}{$\mathbf{S F}$} & $\begin{array}{c}\text { Input image } \\
\mathrm{X}\end{array}$ & 0.6157 & 0.608 & 0.6022 & 0.6557 & 0.2608 & 0.8374 & 0.6123 \\
\hline & $\begin{array}{c}\text { Input image } \\
Y\end{array}$ & 0.4209 & 0.7012 & 0.6519 & 0.5346 & 0.2251 & 0.6198 & 0.64725 \\
\hline & $\begin{array}{c}\text { Fused CXR } \\
\text { COVID-19 } \\
\text { image }\end{array}$ & 0.668 & 0.7944 & 0.7016 & 0.6608 & 0.2958 & 0.9548 & 0.6822 \\
\hline \multirow{3}{*}{ STD } & $\begin{array}{c}\text { Input image } \\
X\end{array}$ & 0.1563 & 0.162 & 0.1921 & 0.1948 & 0.2378 & 0.252 & 0.15 \\
\hline & $\begin{array}{c}\text { Input image } \\
Y\end{array}$ & 0.204 & 0.1496 & 0.2184 & 0.1599 & 0.2109 & 0.19 & 0.1692 \\
\hline & $\begin{array}{c}\text { Fused CXR } \\
\text { COVID-19 } \\
\text { image }\end{array}$ & 0.274 & 0.216 & 0.2544 & 0.2073 & 0.2451 & 0.2548 & 0.2489 \\
\hline
\end{tabular}

Table 6: Evaluation indicator data of various random patients (Source (Input image X, Input image Y) and fused CXR COVID-19 images) 


\section{Table 7 (on next page)}

Fused images evaluation metrics of different algorithms 
1

2

\begin{tabular}{|c|c|c|c|c|c|c|c|}
\hline \multicolumn{2}{|c|}{ Fusion Method } & \multicolumn{7}{c|}{ Evaluation Metrics } \\
\hline \multirow{2}{*}{\begin{tabular}{|} 
Spatial \\
domain
\end{tabular}} & $\begin{array}{c}\text { GFF } \\
\text { (Li et al., 2013) }\end{array}$ & $\mathbf{0 . 5 9 7 8}$ & 3.127 & 42.9419 & 0.4865 & $\mathbf{3 1 . 7 4 8 8}$ & 63.882 \\
\hline $\begin{array}{c}\text { MSA } \\
\text { (Du et al., 2016) }\end{array}$ & 0.3038 & 2.8495 & 28.75 & 0.4829 & 17.0158 & 55.9475 \\
\hline $\begin{array}{c}\text { NSCT+LE } \\
\text { Transform } \\
\text { domain }\end{array}$ & $\begin{array}{c}\text { (Zhu et al., 2019) } \\
\text { NSST+PAPCNN }\end{array}$ & 0.5184 & 2.6052 & 26.0083 & 0.4861 & 31.337 & 75.5464 \\
\hline $\begin{array}{c}\text { Deep } \\
\text { Learning et al., 2018) }\end{array}$ & $\begin{array}{c}\text { Proposed Algorithm } \\
\text { NSCT+CNN-VGG19 }\end{array}$ & 0.5206 & 2.6297 & 25.2629 & 0.4914 & 31.7002 & $\mathbf{7 7 . 6 7 4 8}$ \\
\hline
\end{tabular}

3

Table 7: Fused images evaluation metrics of different algorithms 


\section{Table 8(on next page)}

Comparison with other CNN architectures 
1

Table 8: Comparison with other $\mathrm{CNN}$ architectures

\begin{tabular}{|c|c|c|c|c|}
\hline \multirow{2}{*}{ VGG19 Pre-trained Model } & Tuning Mode & \multicolumn{3}{c|}{ Performance Metrics (\%) } \\
\cline { 3 - 5 } (Abbas et al., 2020) & Shallow (KNN) & 93.42 & 89.71 & 95.7 \\
\cline { 2 - 5 } & Deep Learning (CNN) & 94.59 & 91.64 & 93.08 \\
\hline (Apostolopoulos et al., 2020) & Deep Learning (CNN) & 98.75 & $\mathbf{9 2 . 8 5}$ & 98.75 \\
\hline (Luz et al., 2020) & Deep Learning (CNN) & 75.3 & 77.4 & 50.0 \\
\hline \multirow{2}{*}{ Proposed Framework } & Shallow (KNN) & $\mathbf{9 6 . 9 3}$ & 57.14 & $\mathbf{9 9 . 2}$ \\
\cline { 2 - 5 } & Deep Learning (CNN) & $\mathbf{9 9 . 0 4}$ & 85.71 & $\mathbf{9 9 . 6}$ \\
\hline
\end{tabular}

2 
Figure 1

\section{The Proposed Algorithm Framework}

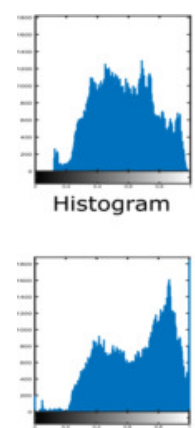

Histogram

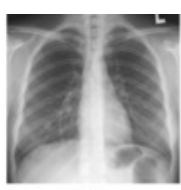

Orignial image $x$ for Patient 1

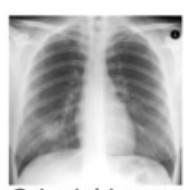
Orignial image
Y for Patient 1

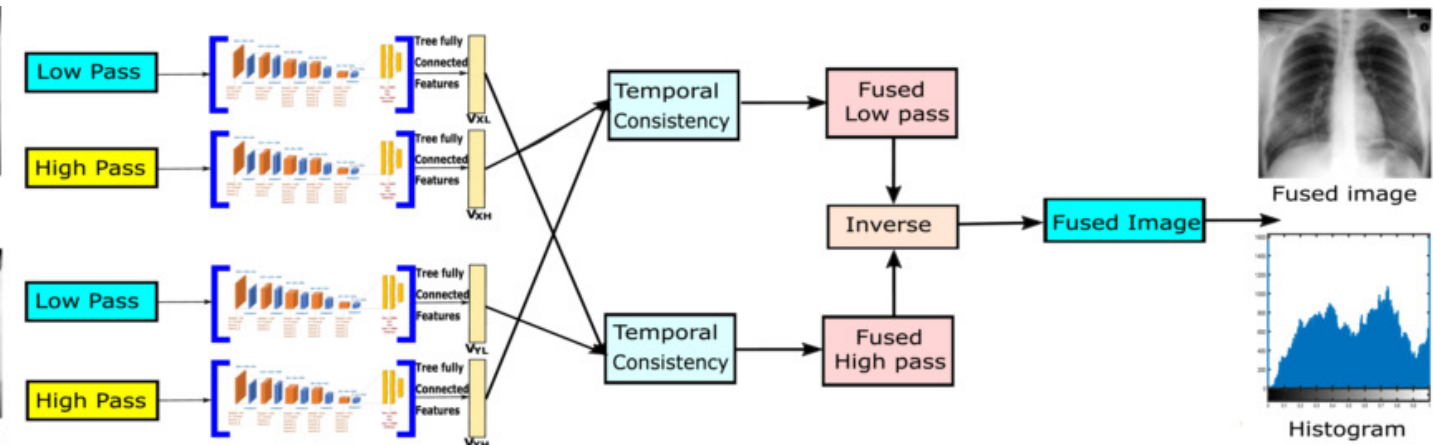


Figure 2

Proposed Perceptual Two Layer Image Fusion using Deep Learning 


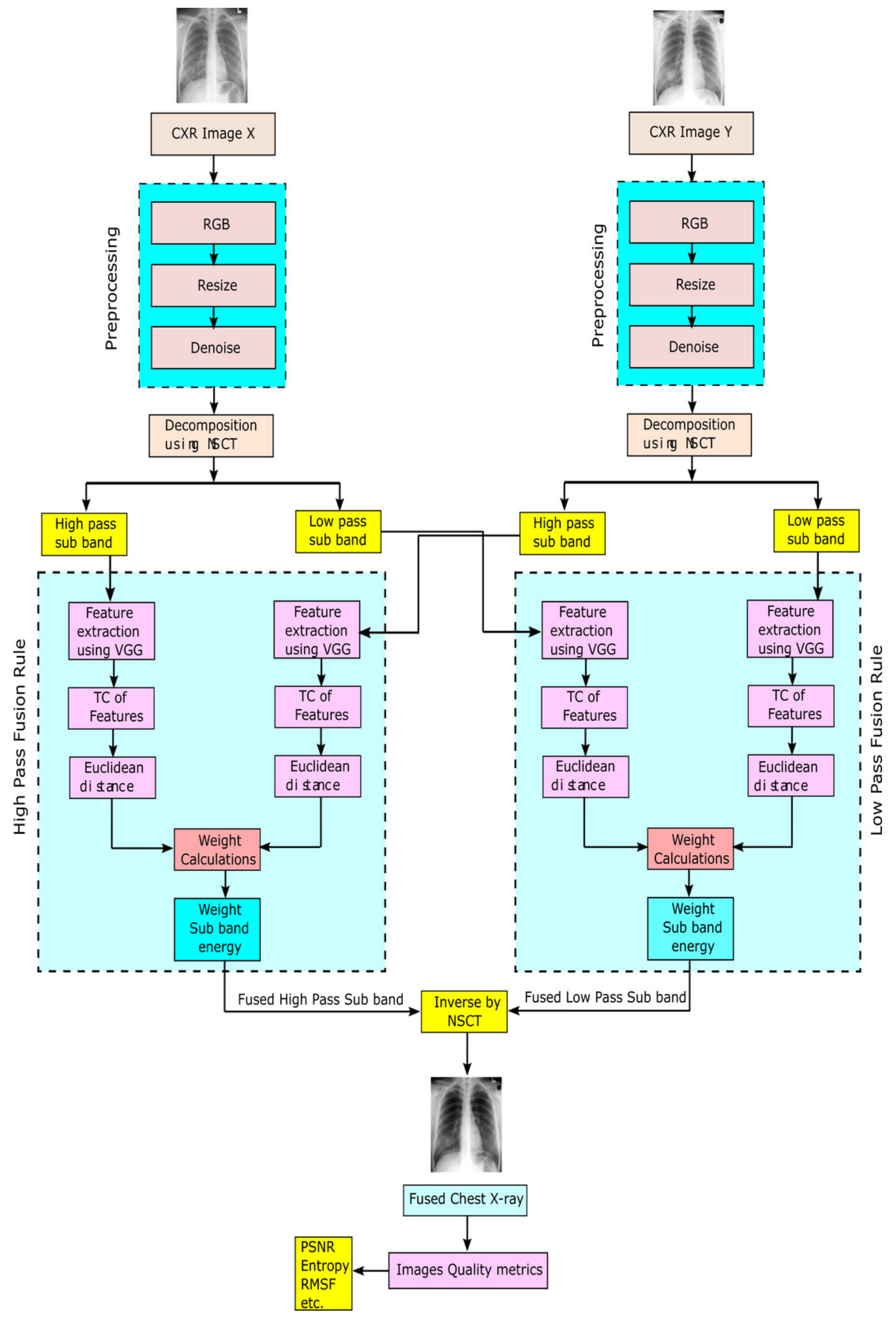


Figure 3

Example of dataset preprocessing steps of raw CXR COVID-19 image
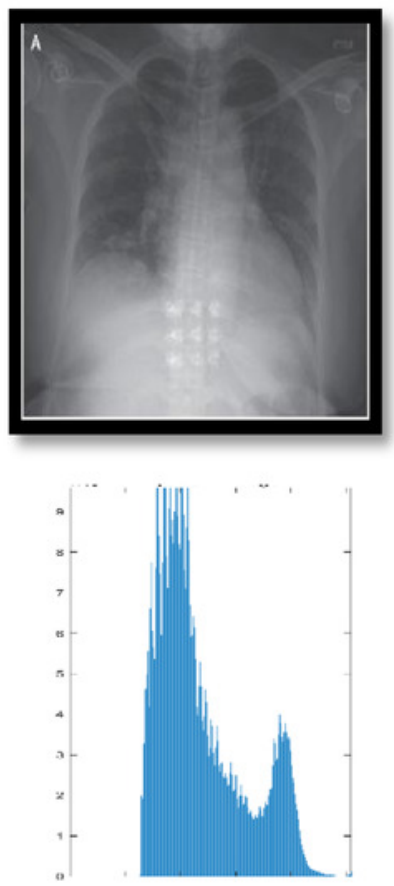

(a) Input CXR COVID-19 image
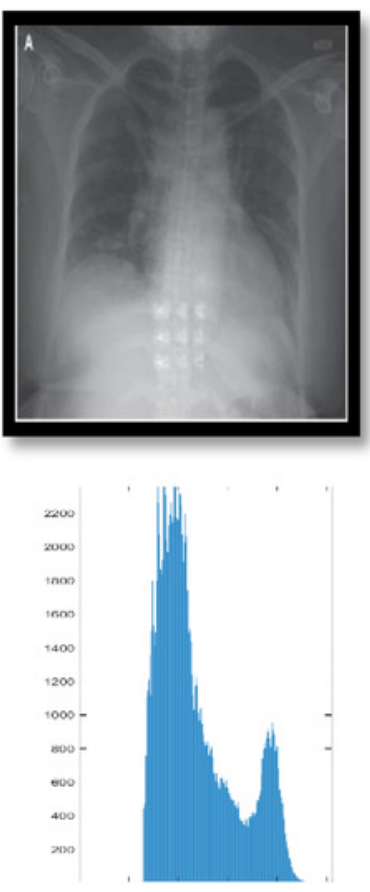

(b) Resized CXR COVID-19 image
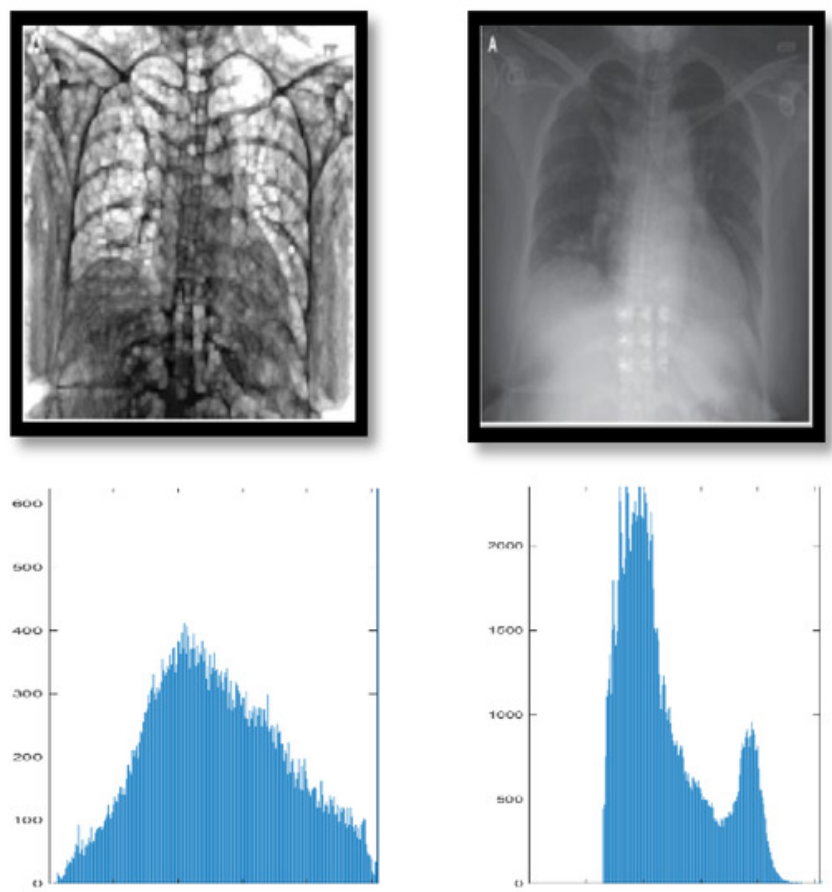

(c) Refined CXR COVID-19 image

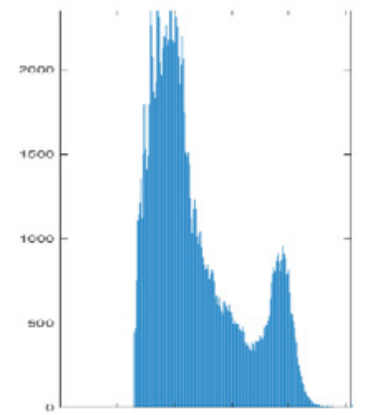

(d) Denoised CXR COVID-19 image 
Figure 4

The NSCT fusion method

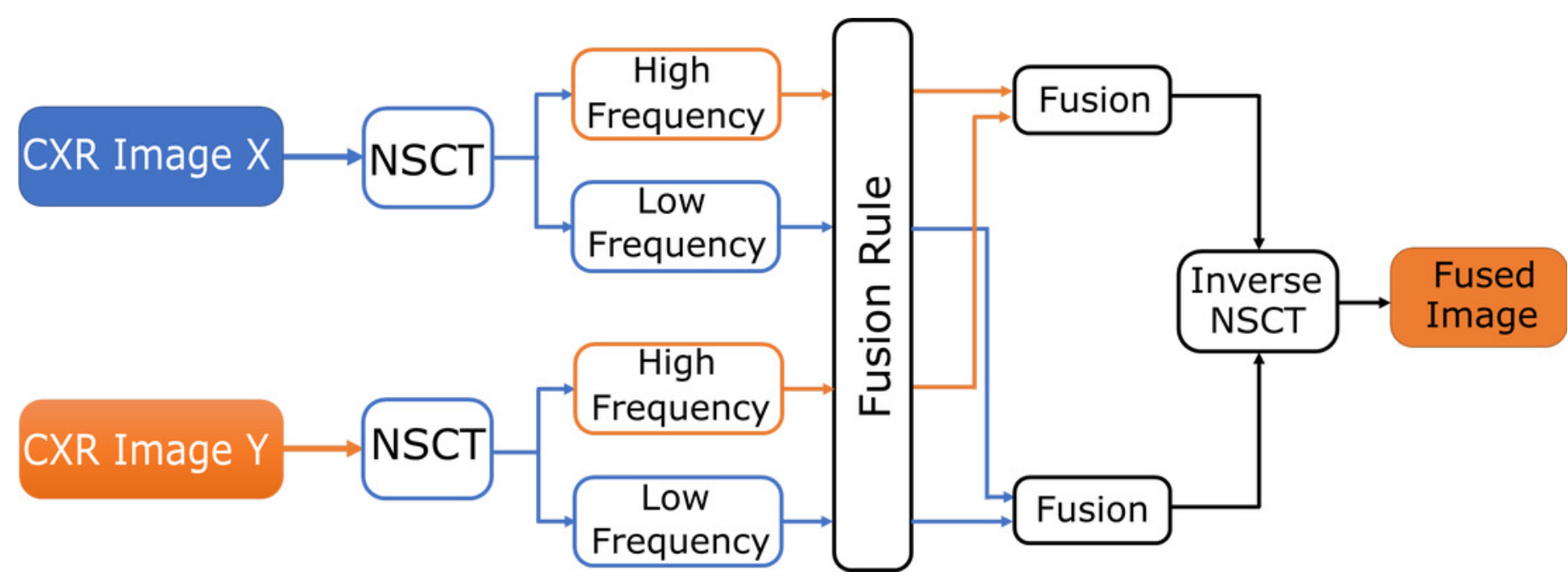




\section{Figure 5}

\section{Blocks Graphical Representation of VGG19}

\section{$224 \times 224 \times 64$}

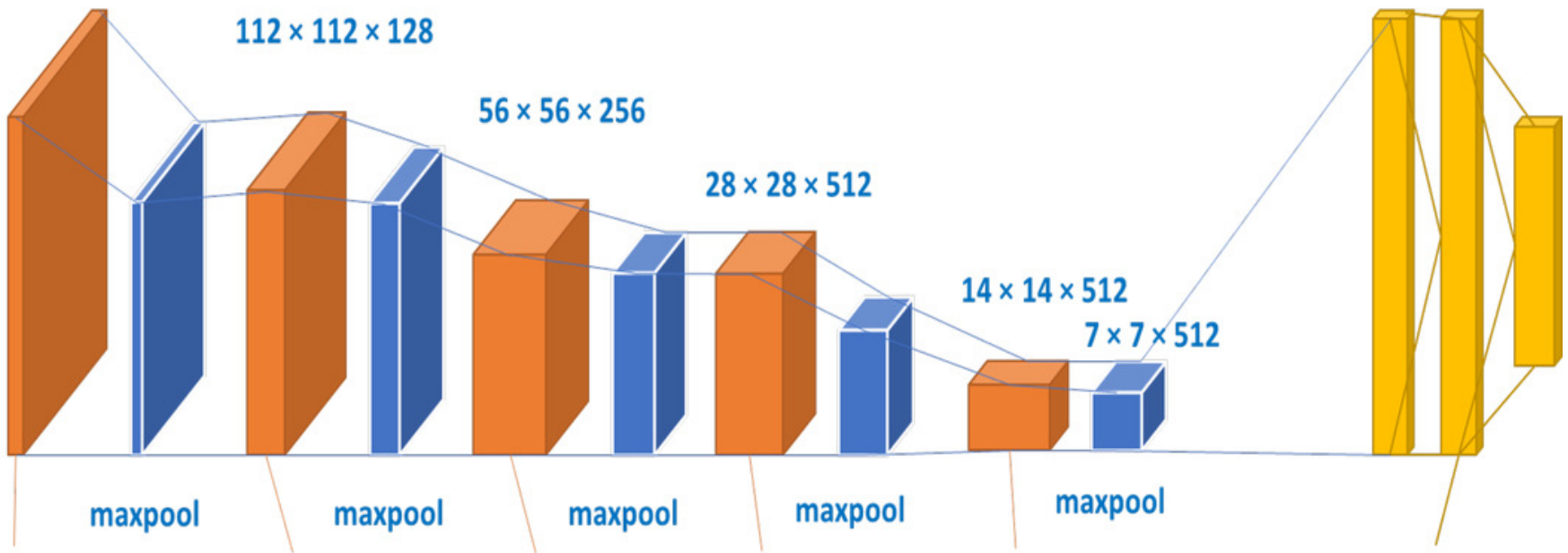

$\begin{array}{lllll}\text { Depth }=64 & \text { Depth }=128 & \text { Depth }=256 & \text { Depth }=512 & \text { Depth }=512 \\ 3 \times 3 \text { Conv } & 3 \times 3 \text { Conv } & 3 \times 3 \text { Conv } & 3 \times 3 \text { Conv } & 3 \times 3 \text { Conv } \\ \text { Conv1_1 } & \text { Conv2_1 } & \text { Conv3_1 } & \text { Conv4_1 } & \text { Conv5_1 } \\ \text { Conv1_2 } & \text { Conv2_2 } & \text { Conv3_2 } & \text { Conv4_2 } & \text { Conv5_2 } \\ & & \text { Conv3_3 } & \text { Conv4_3 } & \text { Conv5_3 } \\ & & \text { Conv3_4 } & \text { Conv4_4 } & \text { Conv5_4 }\end{array}$

Size $=4096$

FC1

$\mathrm{FC2}$

Size $=1000$

Softmax 
Figure 6

(CXR COVID-19 images of a 53-year-old patient with pneumonia after 10 days of infection)
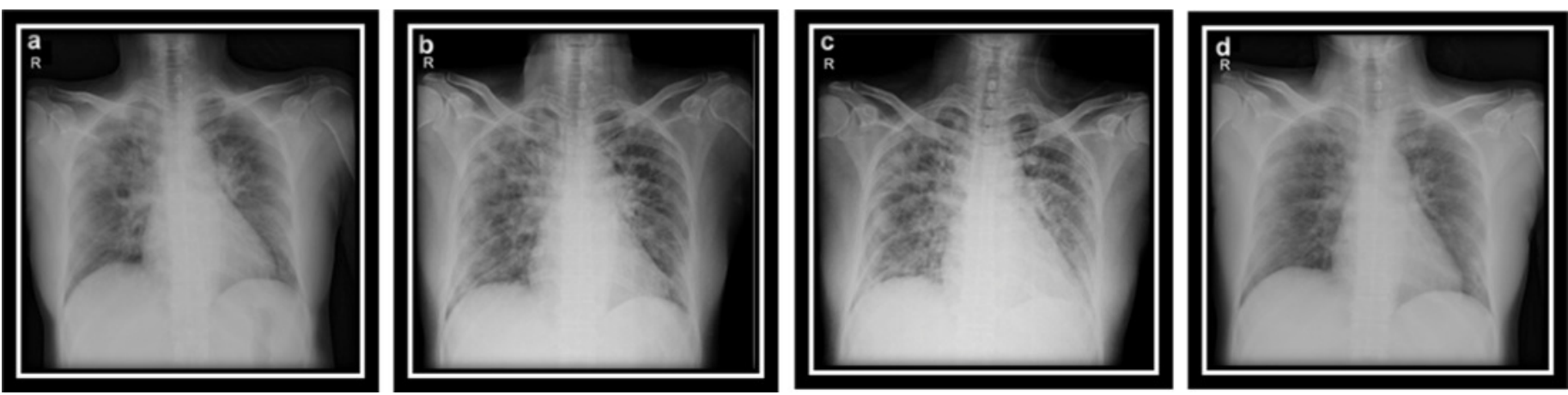
Figure 7

Comparative results of different CNN and the proposed framework CNN-VGG19

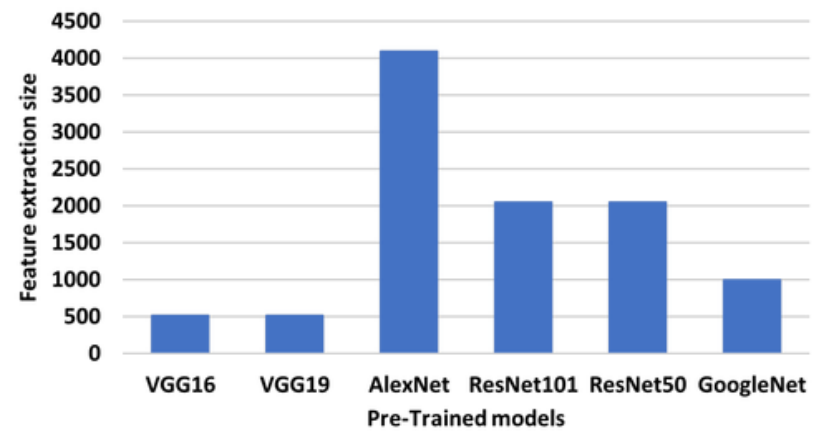

(a)

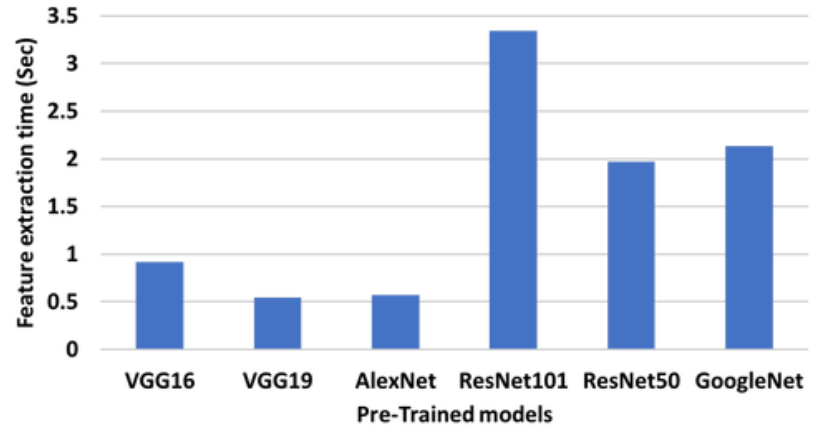

(b) 
Figure 8

Example of a female 50 year's patient (P1) CXR COVID-19 images fusion
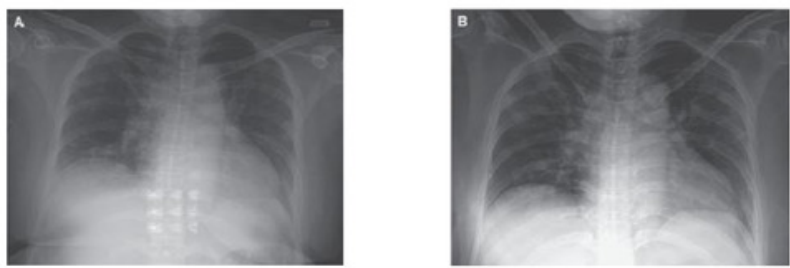

(a) Source of CXR COVID_19 images of a female year's patient

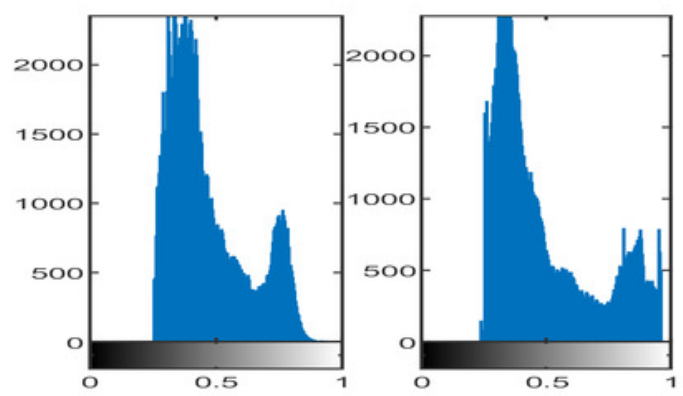

(c) Histogram of CXR source COVID_19 images of a female 52 year's patient

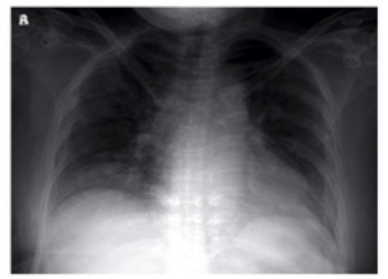

(b) Fused CXR output COVID_19 image of a female 52 year's patient

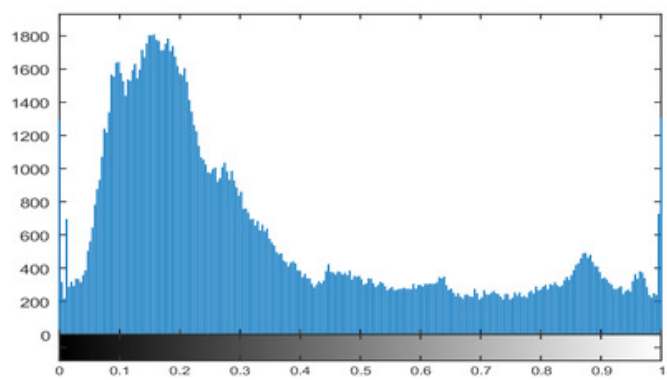

(d) Histogram of fused CXR COVID_19 image of a female 52 year's patient 


\section{Figure 9}

\section{Example of a female 50 year's patient (P1) CXR COVID-19 images fusion}
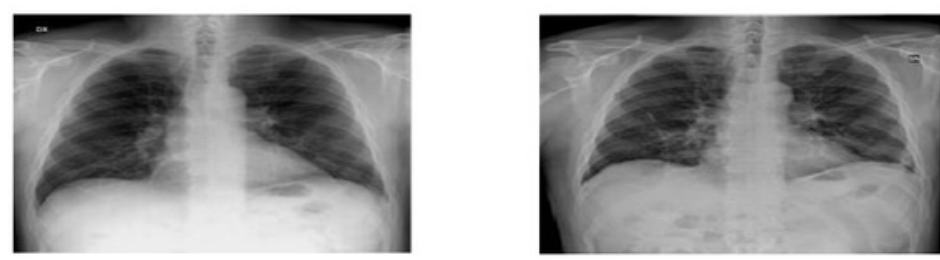

(a) Source of CXR COVID_19 images of a male year's patient

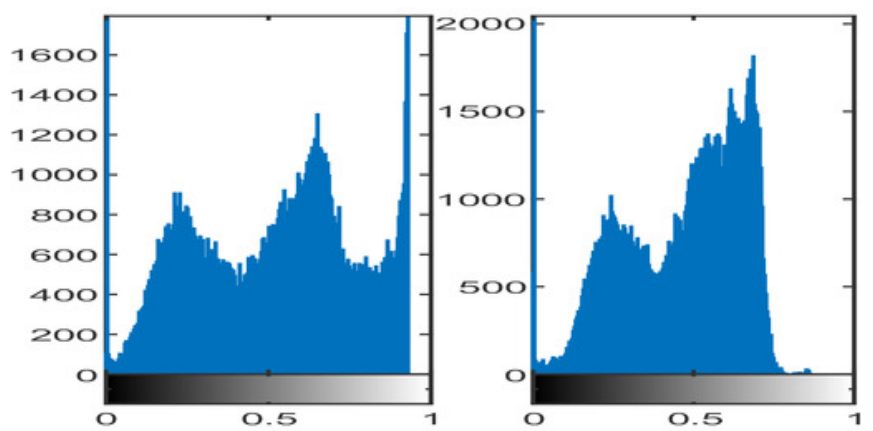

(c) Histogram of CXR COVID_19 source images of a male 67 years patient

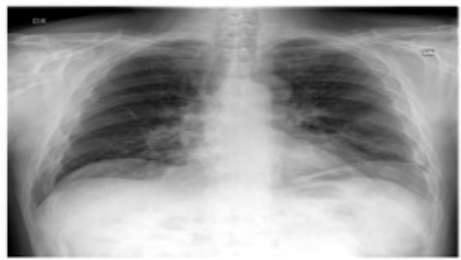

(b) Fused CXR COVID_19 image of a male year's patient

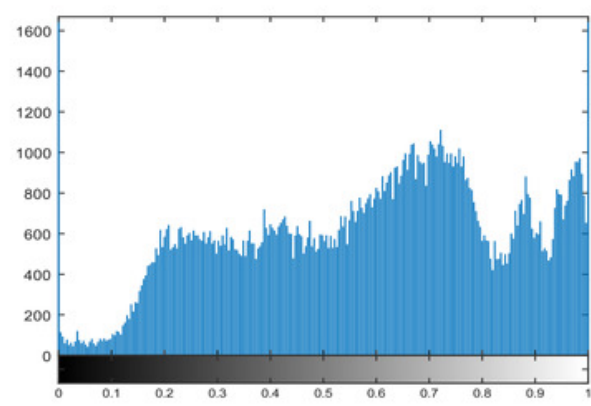

(d) Histogram of fused CXR COVID_19 image of a male 67 years patient 
Figure 10

Comparison between input image $\mathrm{X}$ and fused image of evaluation indicator SF

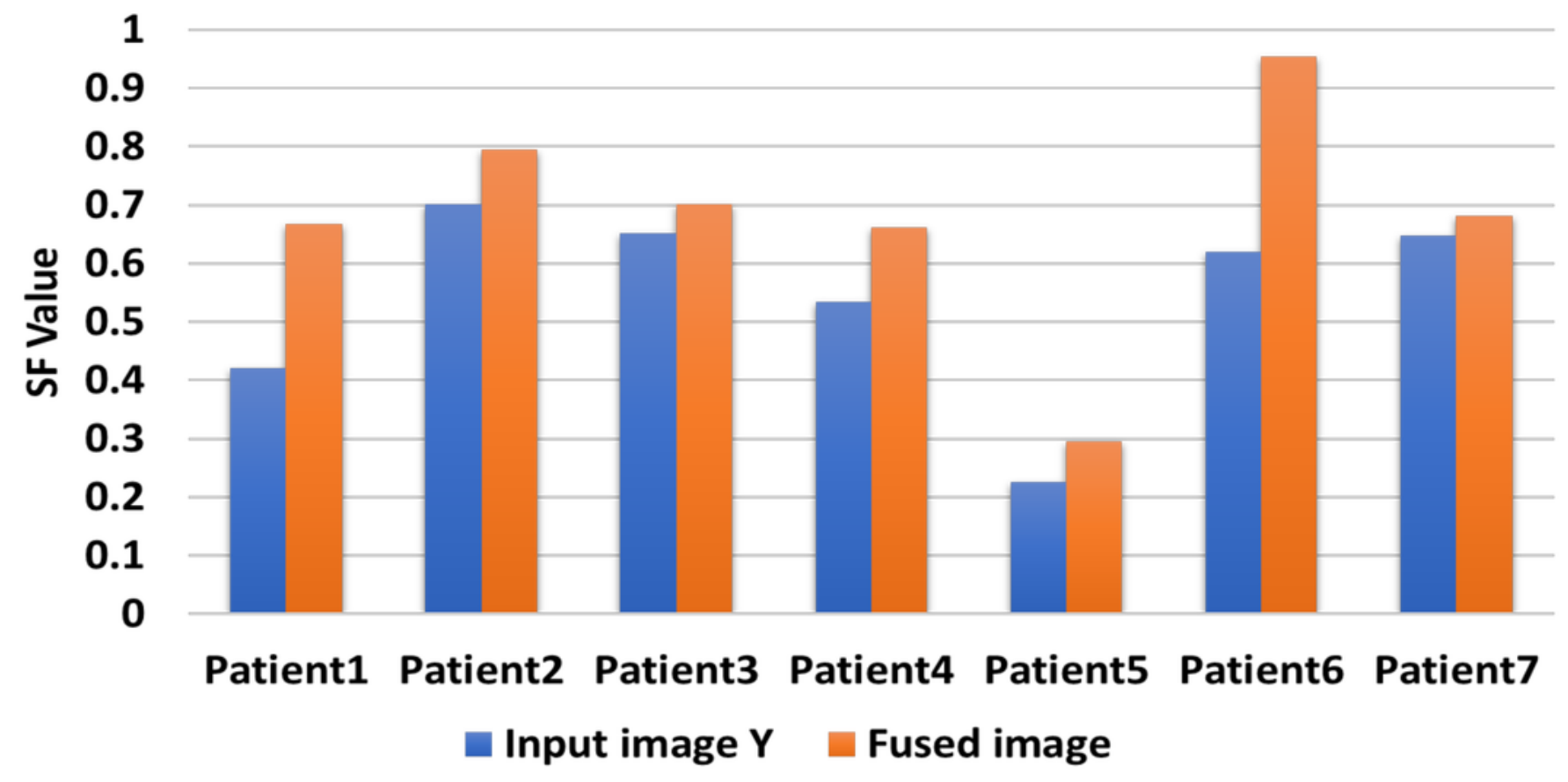


Figure 11

Comparison between input image $Y$ and fused image of evaluation indicator SF

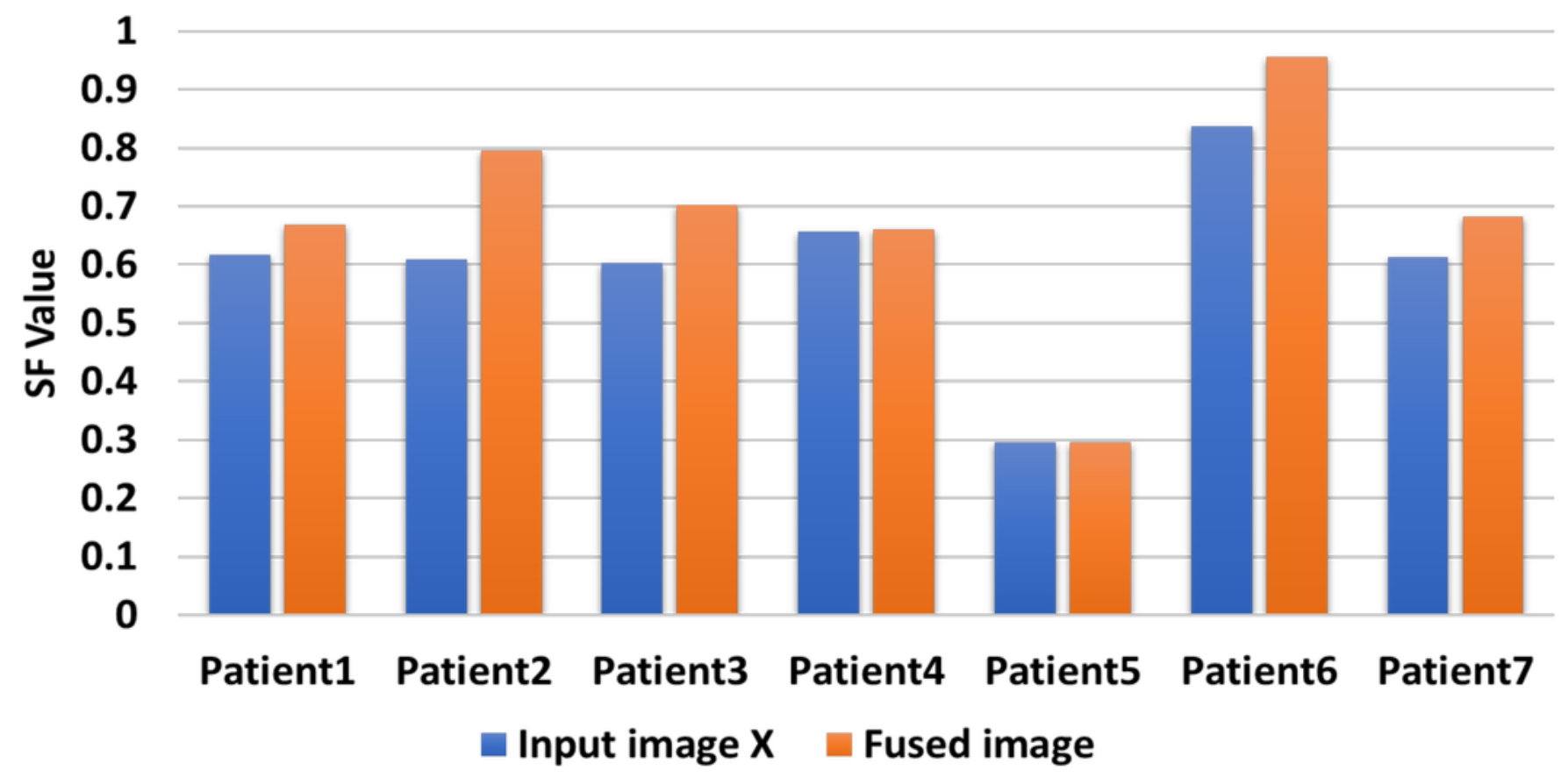




\section{Figure 12}

Comparison between input image $\mathrm{X}, \mathrm{Y}$ and fused image of evaluation indicator STD

0.3

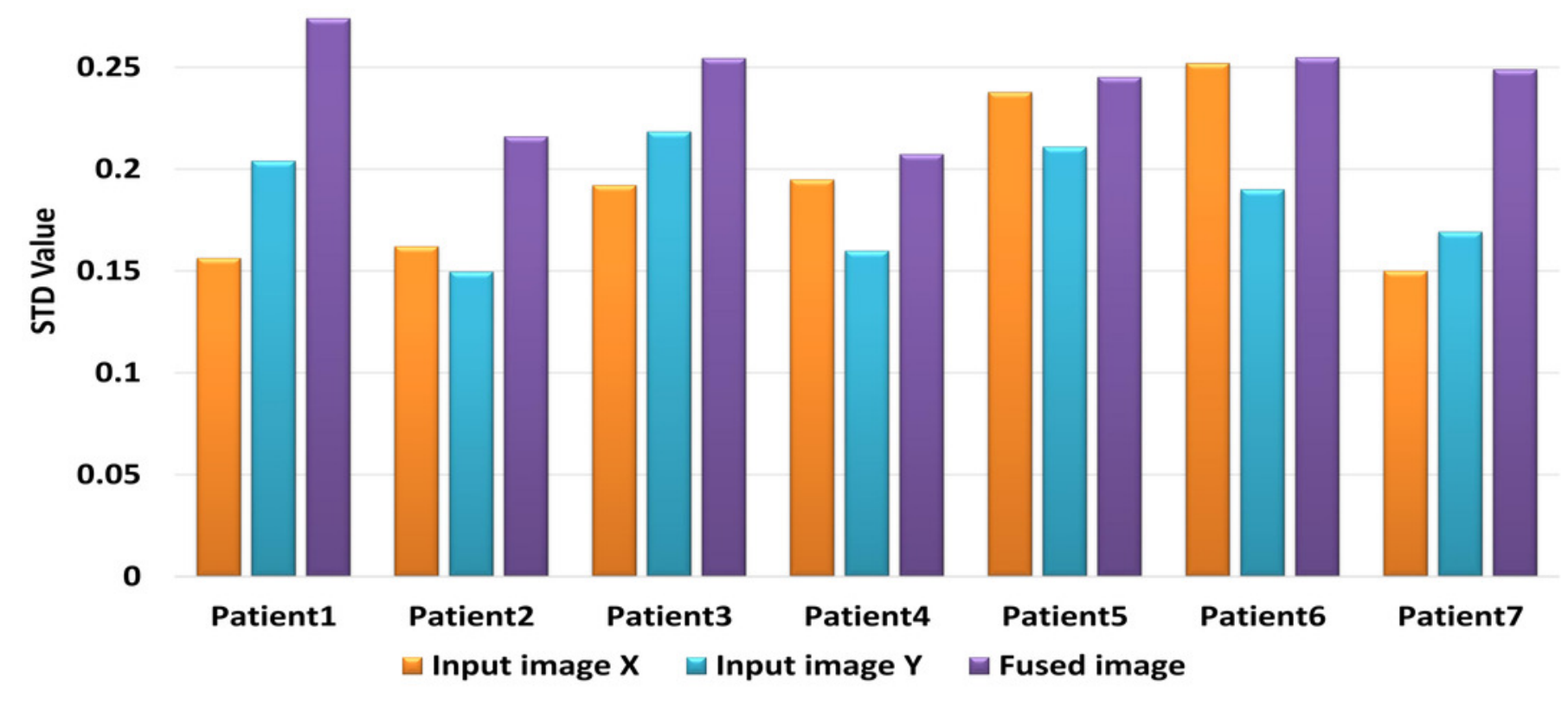


Figure 13

Representation of Evaluation index data of different methods of fused images

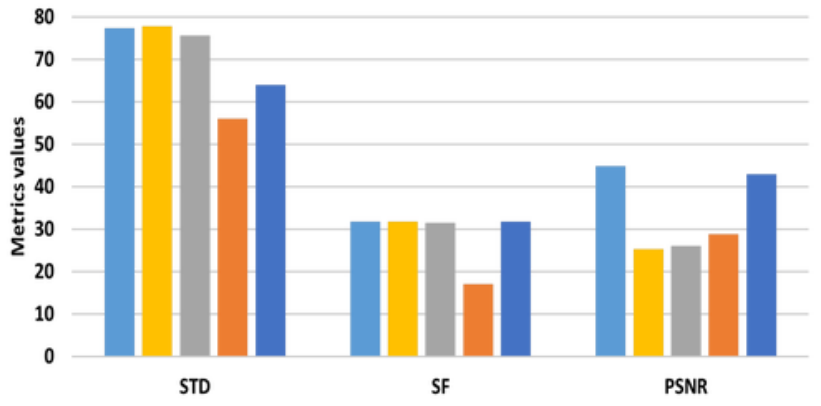

-GFF $\|$ MSA $=$ NSCT+LE $=$ NSST+PAPCNN $\|$ Proposed Algorithm NSCT+CNN-VGG19

(a)

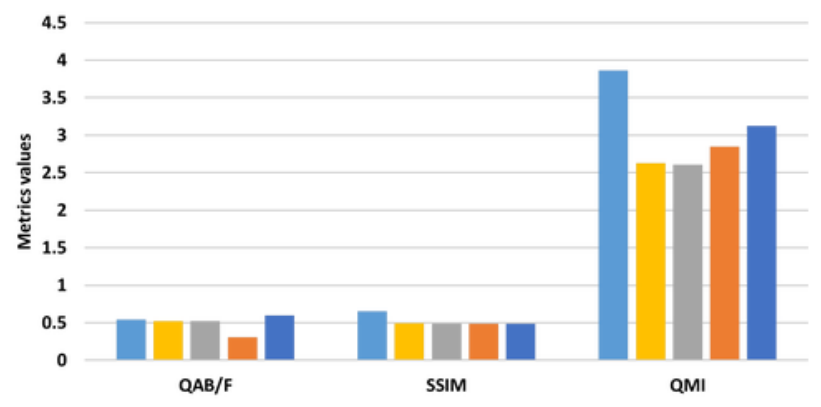

$\|$ GFF $\|$ MSA $=$ NSCT+LE $\|$ NSST+PAPCNN $=$ Proposed Algorithm NSCT+CNN-VGG19 (b) 
Figure 14

Cluster Dendrogram Hierarchy Evaluation of the proposed algorithm with competitive metrics of fused methods for images

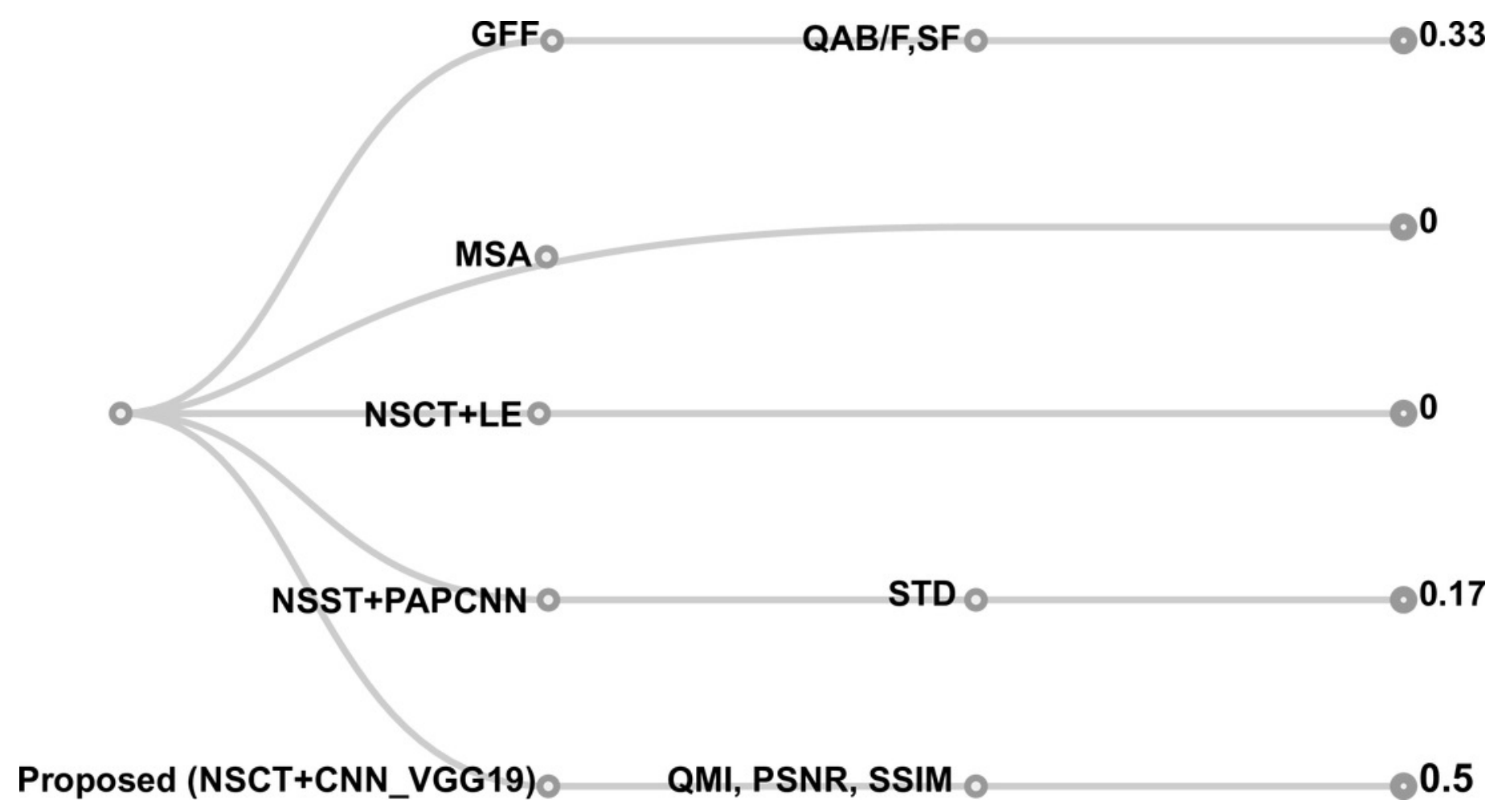




\section{Figure 15}

Confusion matrix of the proposed framework

(a) Confusion matrix of the pre-trained proposed framework using VGG19 for CXR COVID-19 images and (b) Confusion matrix of the pre-trained proposed framework using KNN for CXR COVID-19 images

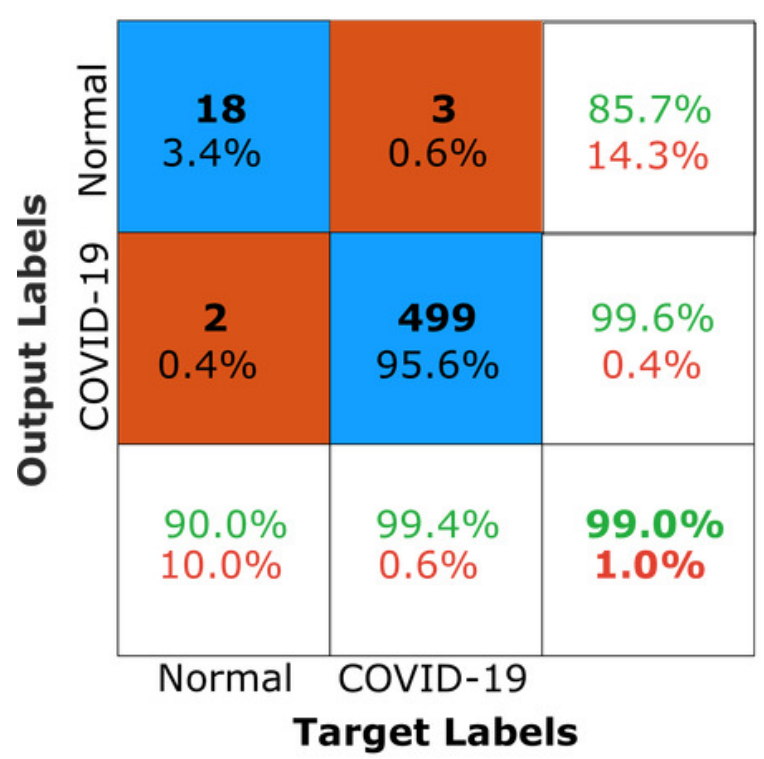

(a)

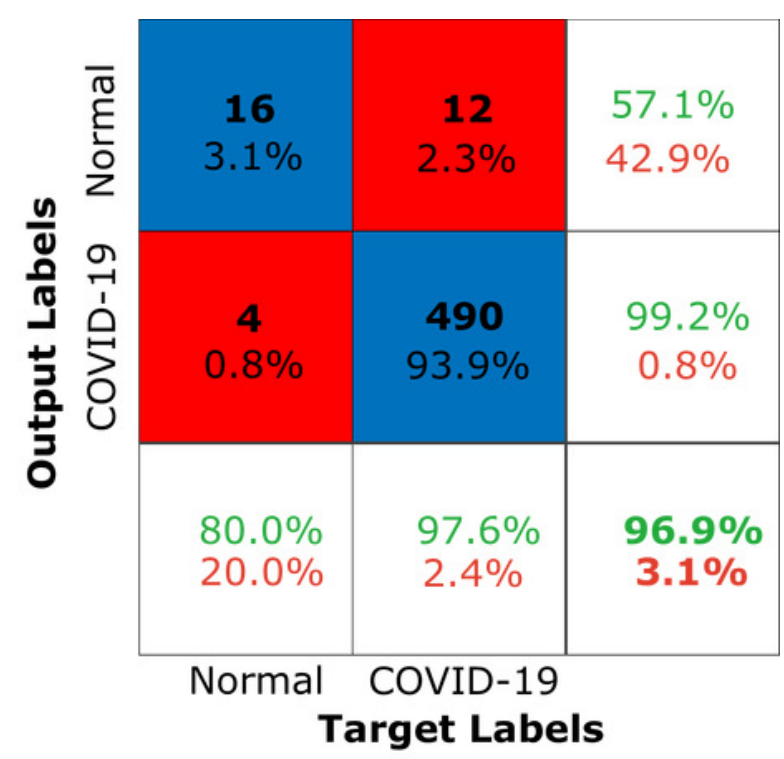

(b) 